\title{
Doppler imaging from artificial data
}

\section{Testing the temperature inversion from spectral-line profiles}

\author{
J.B. Rice ${ }^{1}$ and K.G. Strassmeier ${ }^{2}$ \\ 1 Department of Physics, Brandon University, Brandon, Manitoba R7A 6A9, Canada \\ e-mail: rice@BrandonU.ca \\ 2 Institut für Astronomie, Universität Wien, Türkenschanzstraße 17, A-1180 Wien, Austria \\ e-mail: strassmeier@astro.univie.ac.at
}

Received April 6; accepted August 31, 2000

\begin{abstract}
We present extensive numerical tests of our temperature-based image reconstruction code TempMap. Two test cases that represent a rapidlyrotating low-inclination star and a moderately-rotating high-inclination star are used for the forward problem. Tests are then made to recover the original input map and include data errors and input-parameter errors. The former include variations of photon noise, continuum displacement, continuum slopes, scattered light in the spectrograph, and phase gaps with and without continuum information. The input-parameter tests include the confusion in the recovery of hot versus cool spots, uncertainties of atmospheric parameters such as radialtangential macroturbulence and gravity, and the influence of line strength and line damping. In general, we find that the geometric information is less dependent on photon noise and on input errors than the temperature information. Our test inversions also show that, at the $v \sin i$ of these test cases, no significant gain in image quality is achieved once $S / N$ of $300: 1$ is surpassed.
\end{abstract}

Key words: stars: imaging — stars: activity — line: profiles — techniques: spectroscopic

\section{Introduction}

Doppler imaging is an elaborate observational and computational technique similar to medical tomography wherein a series of high-resolution spectral line profiles are inverted into an "image" of the stellar surface. A complex computer code - hereafter called TEMPMAP - was developed by Rice et al. (1989) for Ap stars and subsequently was applied to spectral-line data for numerous cool stars in a

Send offprint requests to: K.G. Strassmeier series of papers (e.g. Strassmeier \& Rice 2000). With every application, our code continuously evolved and features were frequently added or removed and tested. The actual test results are extensive and could not be accommodated within the "regular" astronomical papers. Following suggestions from previous referees who had questions about the behavior of Doppler imaging under less than ideal conditions, we felt now that the time was ripe to present an update of the TEMPMAP code and document the reliability (and weaknesses) of our code through a series of tests on artificial data.

The basic concepts of TEMPMAP were layed out in the original paper by Rice et al. (1989) and Doppler imaging with it was reviewed, among others, by Piskunov \& Rice (1993), Rice (1996), and Strassmeier (2000). A brief description of the new features of TEMPMAP can be found in previous papers, e.g., Rice \& Strassmeier (1998) and references therein. In the present paper, we will concentrate on the test results.

Other codes were developed in parallel and include the early efforts of Vogt et al. (1987) and Piskunov (1990). Many successful applications appeared in the literature and a comparison of these two codes with an early version of TEMPMAP was made using EI Eri as a test star (Strassmeier et al. 1991). Generally, the comparison was satisfactory given the state of Doppler imaging at that time. In 1990, Collier Cameron (1992) introduced his code which, in the meantime, has experienced numerous upgrades and successful applications to AB Dor (e.g. by Unruh et al. 1995). Also in that year, Kürster et al. (1990) presented an inversion code based on the CLEAN algorithm and later conducted extensive testing (Kürster 1993) along with an application to AB Dor. In 1992, the French group around M. Semel introduced their mapping version (Donati et al. 1992) which had been also continuously upgraded and tested since then 
Table 1. Adopted stellar parameters for the forward calculations of profiles

\begin{tabular}{lll}
\hline & Case 1 & Case 2 \\
\hline $\log g$ & 4.0 & 4.0 \\
$T_{\mathrm{phot}}$ & 5000 & 5000 \\
$v_{\mathrm{e}} \sin i$ & 28 & 41 \\
Inclination $i$ & 65 & 30 \\
$v_{\mathrm{e}}$ & 31 & 82 \\
Microturbulence & 1.0 & 1.0 \\
Macroturbulence & 3.5 & 3.5 \\
Abundance & solar & solar \\
\hline
\end{tabular}

(e.g. Donati 1999). Two more french groups presented papers on new computer codes, Jankov \& Foing (1992) and Joncour et al. (1994). Testing was presented by the former while two applications were presented by the latter. Most recently, Berdyugina (1998) presented a code based on the Occamian principle and successfully applied it to the RS CVn star II Pegasii (Berdyugina et al. 1998).

\section{Summary of TempMap features and test properties}

\subsection{Line profile inversion with a penalty function}

TEMPMAP recovers the surface temperature distribution from the integral equation that relates the distribution of surface temperature to the observed line profile and light curve variations. Local line profiles are computed from a numerical solution of the equation of transfer with 72 depth points from the grid of model atmospheres published by Kurucz (1993). These model atmospheres are precalculated assuming LTE. The local line profile for each small surface segment is obtained from the grid of profiles by interpolation to match the local effective temperature.

The effects of noise in the data are controlled by a penalty function, or regularizing functional, that prevents the "overinterpretation" of information contained in the line profiles and the light curve. TEMPMAP incorporates a choice between using a maximum entropy penalty function in solving the inverse problem, or using a Tikhonov penalty function. The form taken by the maximum entropy penalty function is

$f_{\text {en }}=\sum_{i}$ Flux $_{i} \ln \left(\operatorname{Flux}_{i}\right)$

Here Flux is approximated by $\omega T^{4}$ with $\omega$ as an arbitrary constant and $T$ as the local effective temperature normalized by the average effective temperature over the surface of the star. The Tikhonov penalty function is usually represented as

$f_{\mathrm{t}}=\int_{-\pi}^{\pi} \int_{-\pi / 2}^{\pi / 2}\left[\left(\frac{\partial \tau}{\partial \phi}\right)^{2}+\left(\frac{\partial \tau}{\partial l}\right)^{2}\right] \mathrm{d} \phi \mathrm{d} l$
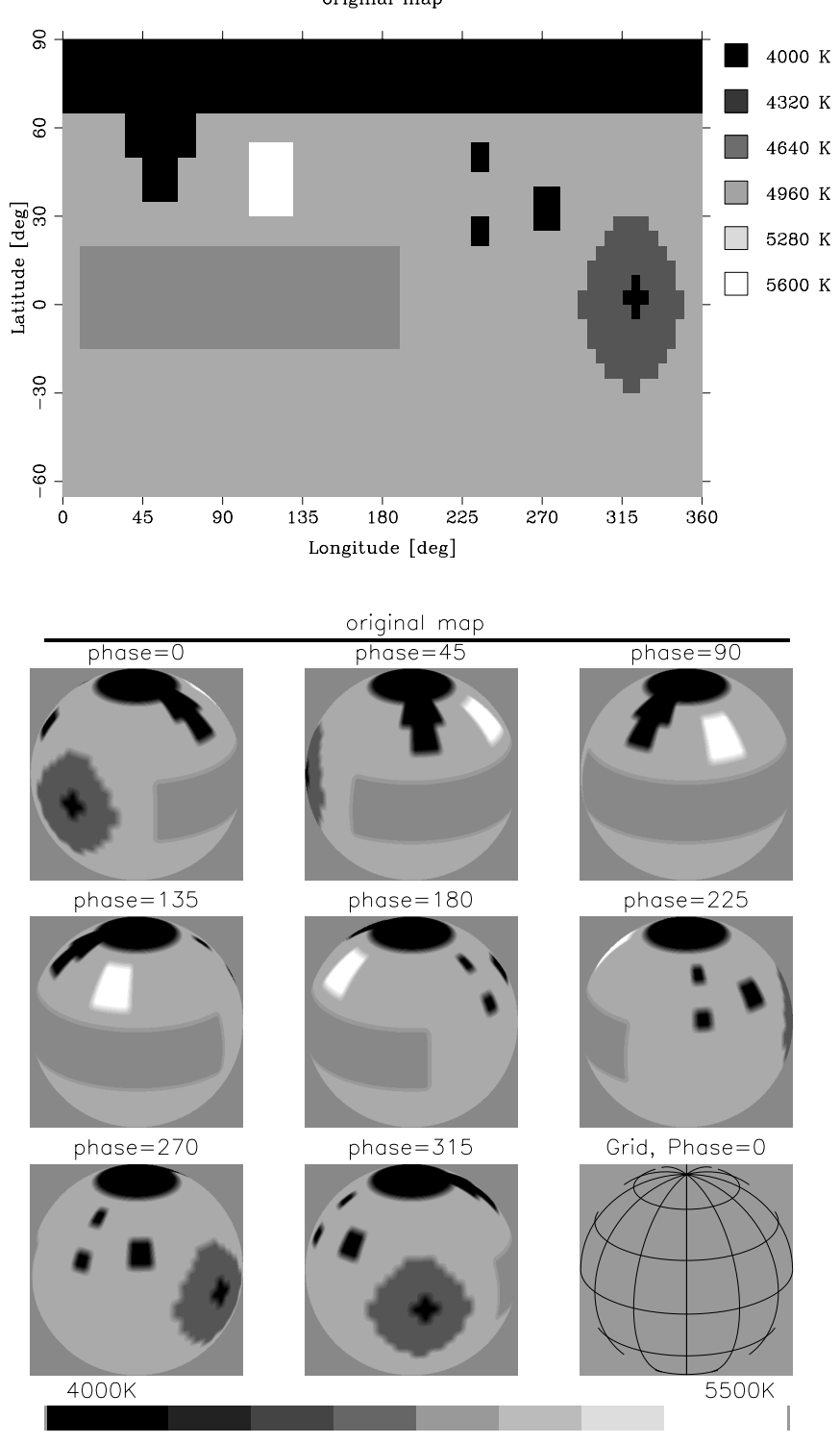

Fig. 1. Artificial input map for the forward data for Case 1 (Case 2 is identical but with different inclination and rotational broadening). The graph shows the input map in Mercator projection (top) and in spherical projection at eight rotation phases (bottom)

where $\phi$ represents latitude on the stellar surface and $l$ is a linearized measure of longitude. (See Piskunov \& Rice 1993 for further description). In practice, the choice between these has little significance because normally only small weighting is given to the penalty function when the noise problem is not serious.

Continuum light variations and their zero point in two bandpasses are also employed by the mapping routine to further constrain the solution. 


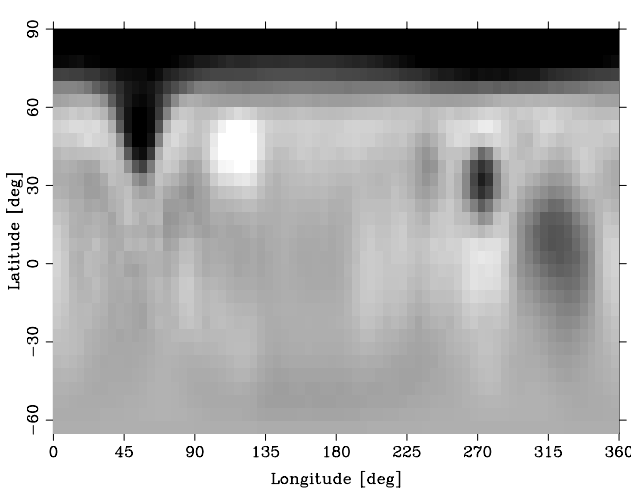

$$
\mathrm{S} / \mathrm{N}=3000
$$
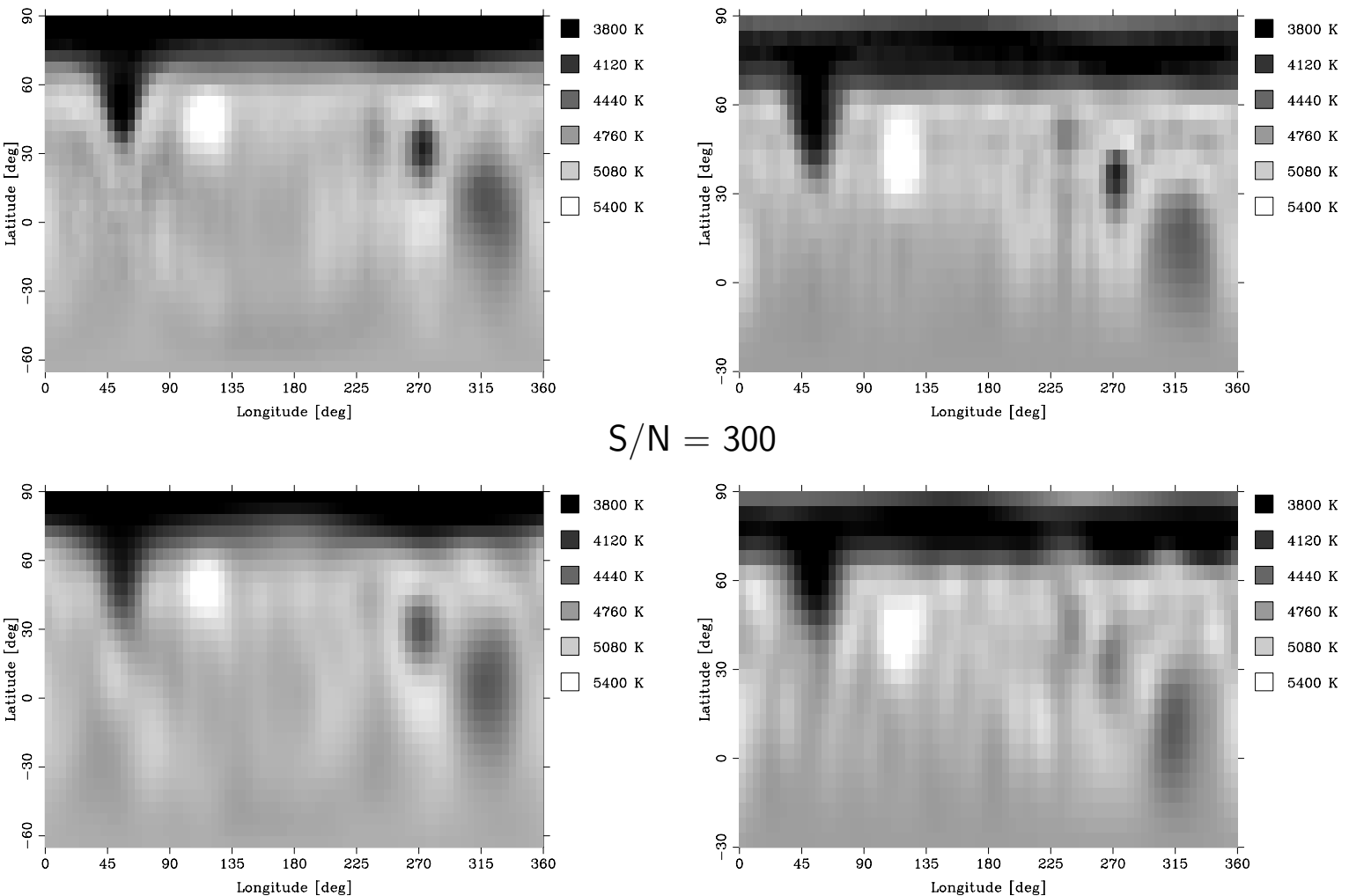

$\mathrm{S} / \mathrm{N}=300$
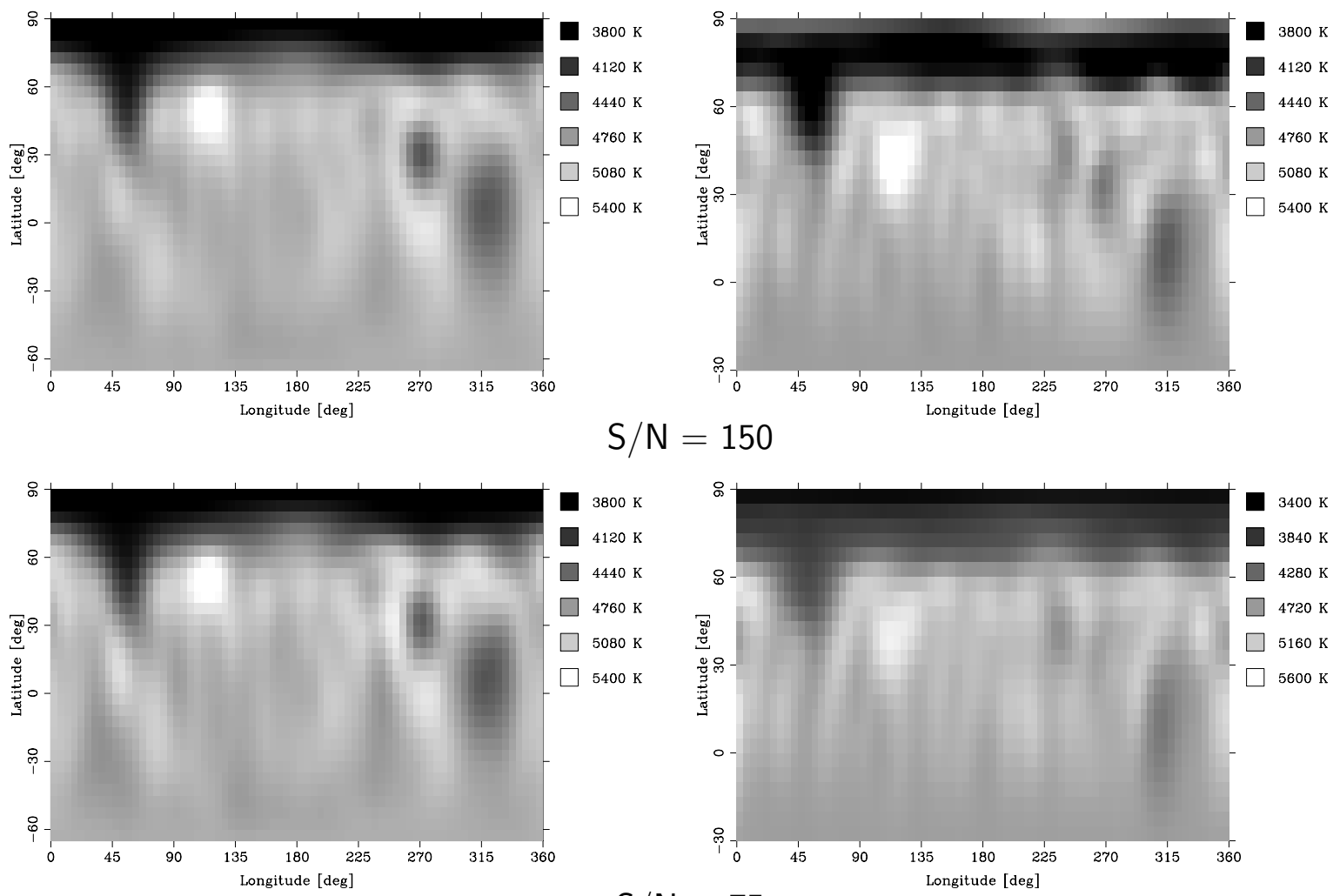

$$
\mathrm{S} / \mathrm{N}=150
$$
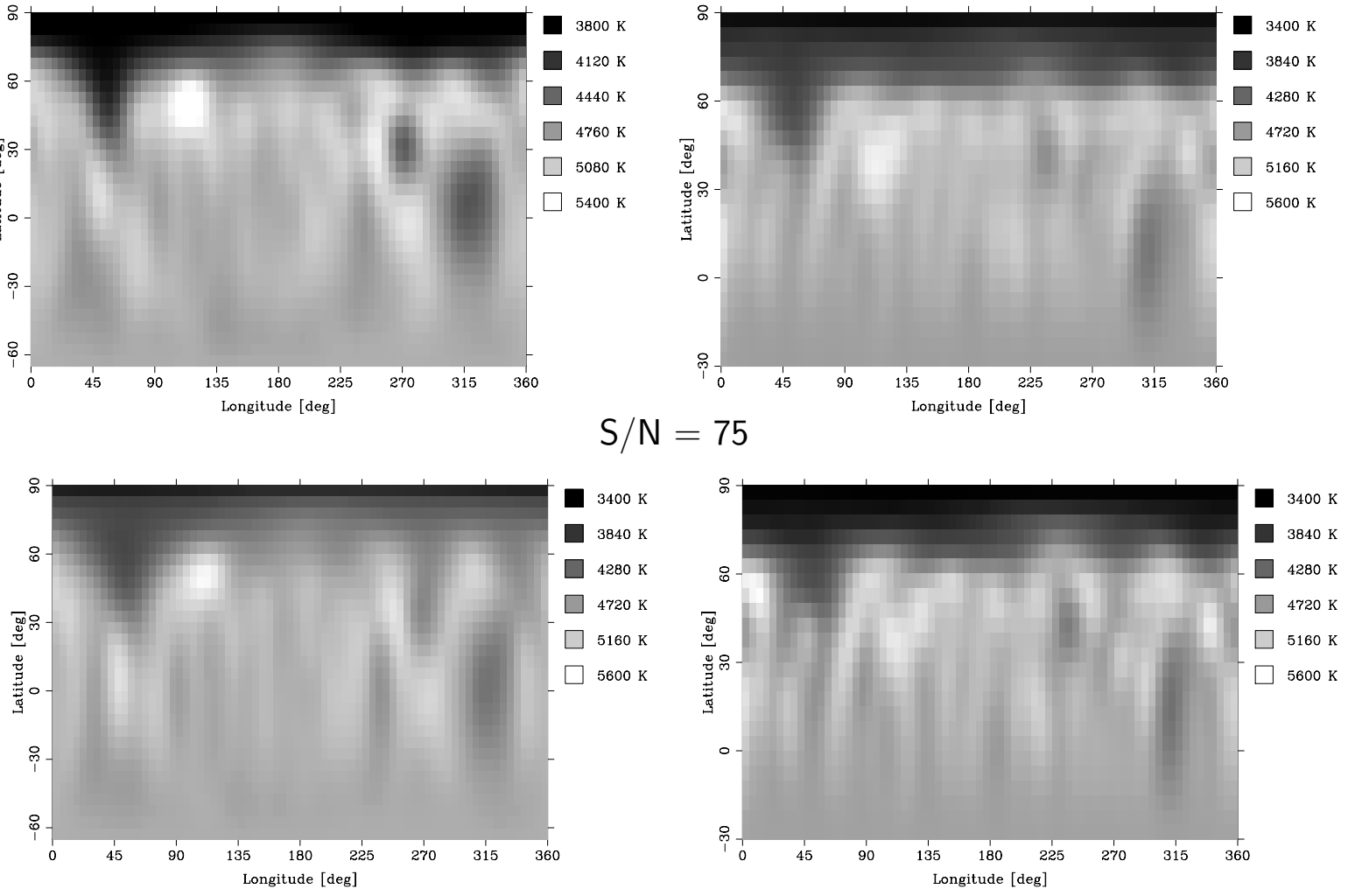

Fig. 2. Examples of recovery using a Tikhonov penalty function at various $S / N$ ratios. From top to bottom: $S / N=3000: 1$, 300:1, 150:1, and 75:1. The images in the left column are for Case 1, the images in the right column for Case 2. Note that there is no significant difference between the reconstructions once a $S / N$ ratio of approximately 300:1 is surpassed. The reconstruction from $S / N=600$ (not shown) is practically identical to the $S / N=3000$ case 

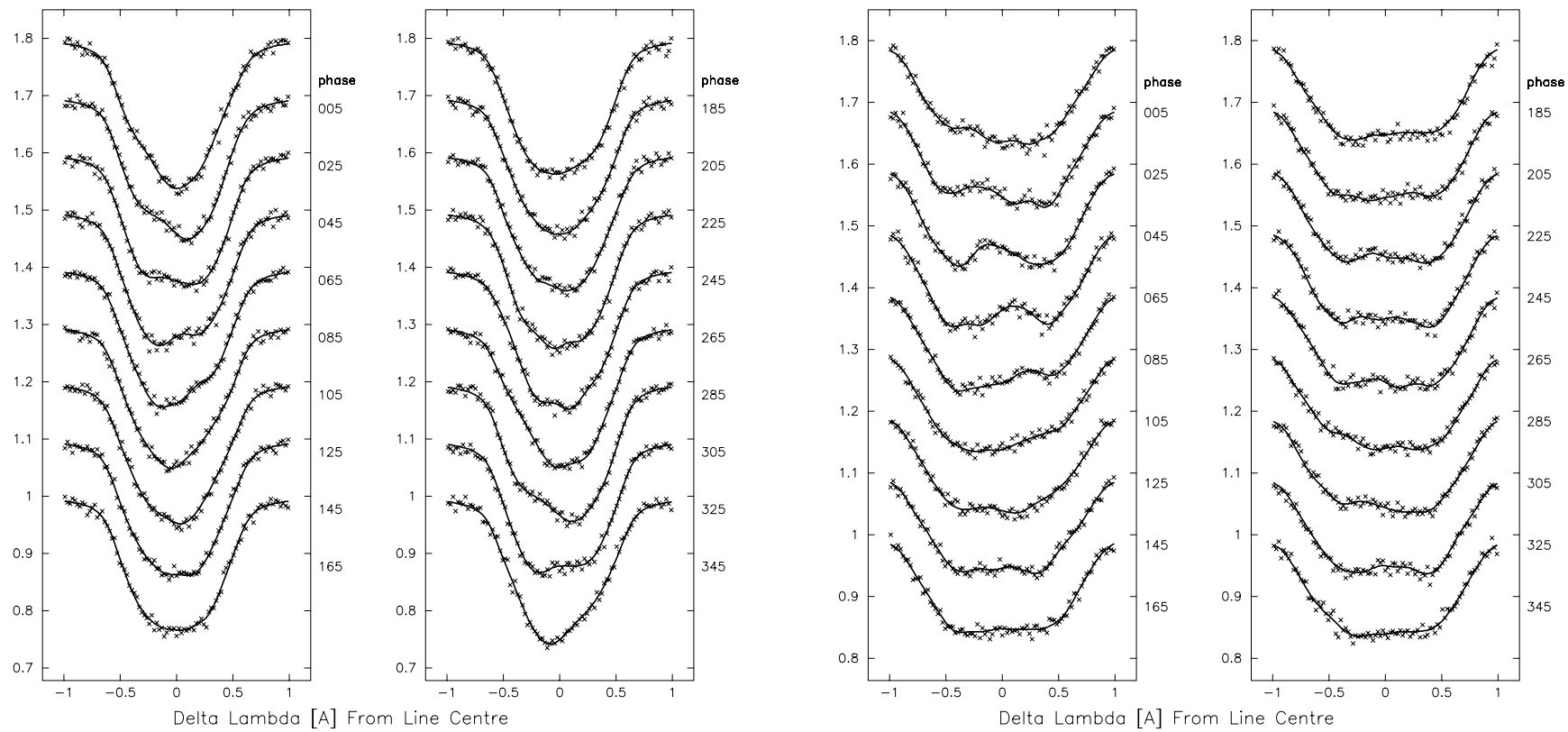

Fig. 3. The artificial spectral line data (crosses) and their respective fits (lines) for $S / N=150: 1$. Left two panels: Case 1 , right: Case 2
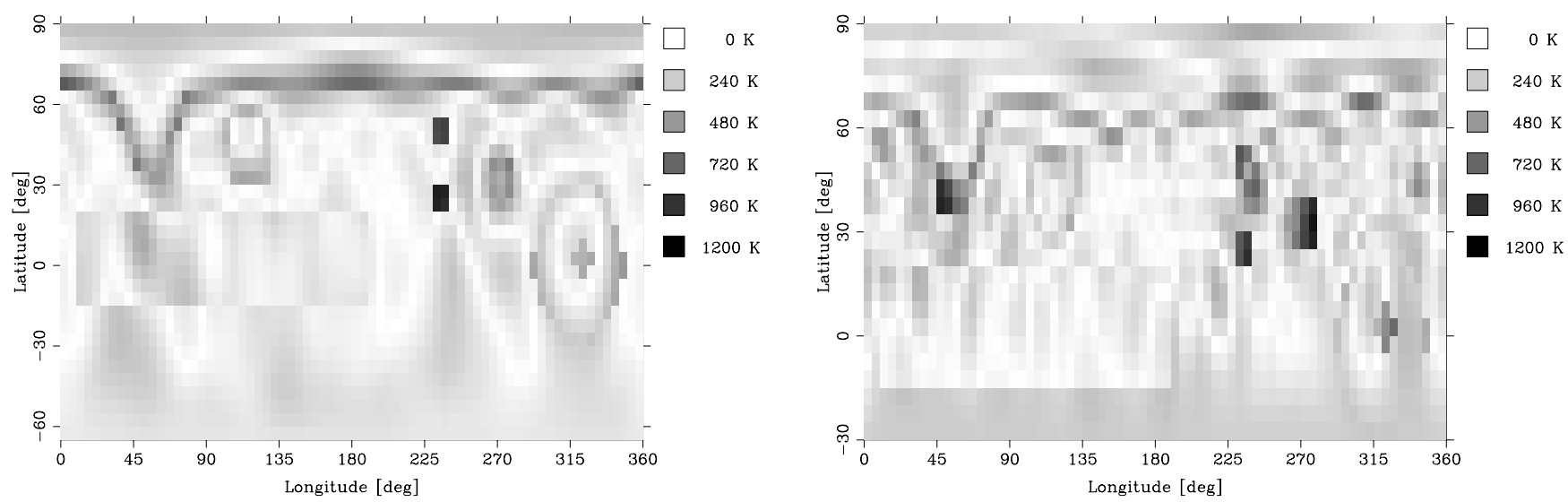

Fig. 4. The difference maps input-output for $S / N=150: 1$. Left: Case 1, right: Case 2. The grey scale indicates the temperature difference in Kelvin. Note that white regions indicate no difference, and black regions indicate maximum difference. These maps can also be viewed as "sensitivity" maps for a given input map; the most sensitive areas being the darkest regions

\subsection{Artificial test data}

There are two case situations, i.e. two hypothetical stars resembling typical stellar applications. The stellar parameters for these test stars, called Case 1 and Case 2, are listed in Table 1 . They differ only by their rotational velocity and inclination of the rotational axis. We excluded the case of a slow rotator, i.e. $v \sin i<20 \mathrm{~km} \mathrm{~s}^{-1}$, from the tests because that was an issue in a previous paper (Strassmeier \& Rice 1998a).

Forward calculations are performed for both cases with the appropriate subroutines of TEMPMAP and used the parameters of the FeI line at $6411 \AA$, one of the most widely used spectral line for Doppler imaging. The atomic line parameters were adopted from the Kurucz (1993) line list; with the logarithmic transition probability $(\log g f)$ set to -0.35 , a lower excitation potential of $3.654 \mathrm{eV}$, and damping constants that are computed from the classical damping formula from Unsöld (1955) for this forward calculation (more extensively discussed later in Sect. 4.3). Note that the literature provides various values for $\log g f$ of this line. The VALD database (Piskunov et al. 1995; Kupka et al. 1999) lists -0.595, Kurucz (1993) listed -0.820 , Lambert et al. (1996) adopted -0.66, and King (1999) obtained -0.717 . We have used a larger value than is given in most of the tables. That is because we have found in the past that we needed a larger value even when all the blending lines we thought could be there were taken into account.

The grid spacing on the stellar surface was $5^{\circ} \times 5^{\circ}$, giving a total of 2592 pixels visible if the star is at an 
$\Delta \mathrm{I}=+0.005$, abund.$=$ const

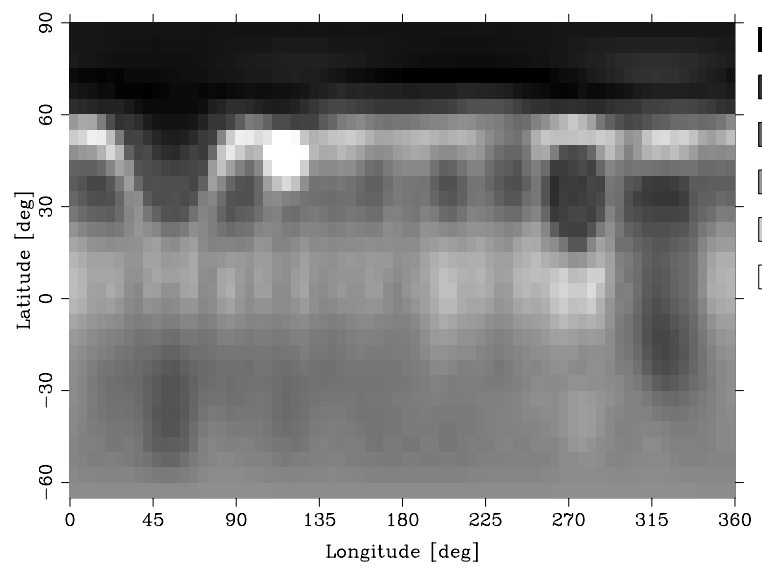

$\Delta \mathrm{I}=-0.005$, abund.$=$ const

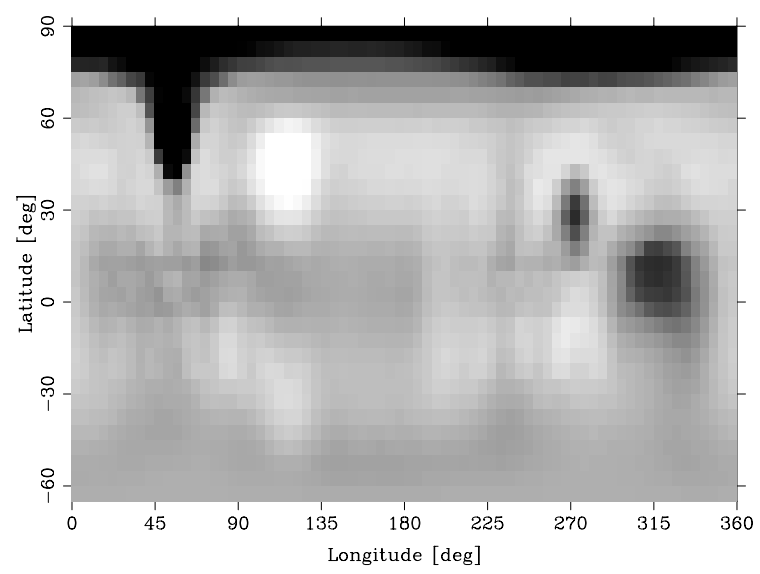

Photometric Region $=5500$

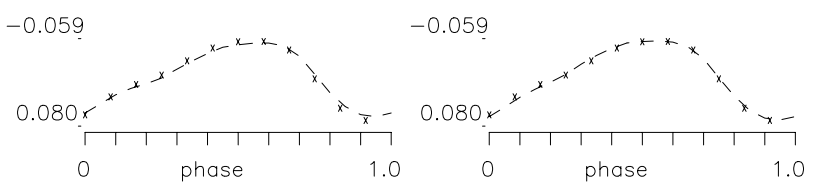

Photometric Region $=7900$

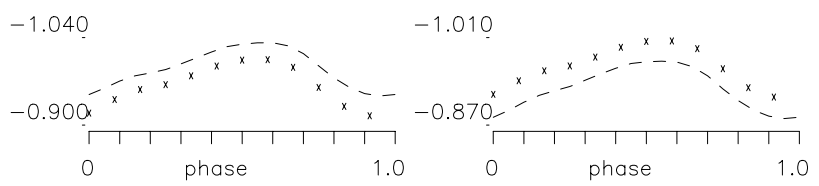

\section{Continuum shift:}

$$
\Delta \mathrm{I}=+0.005, \text { abund } .=\text { adjusted }
$$

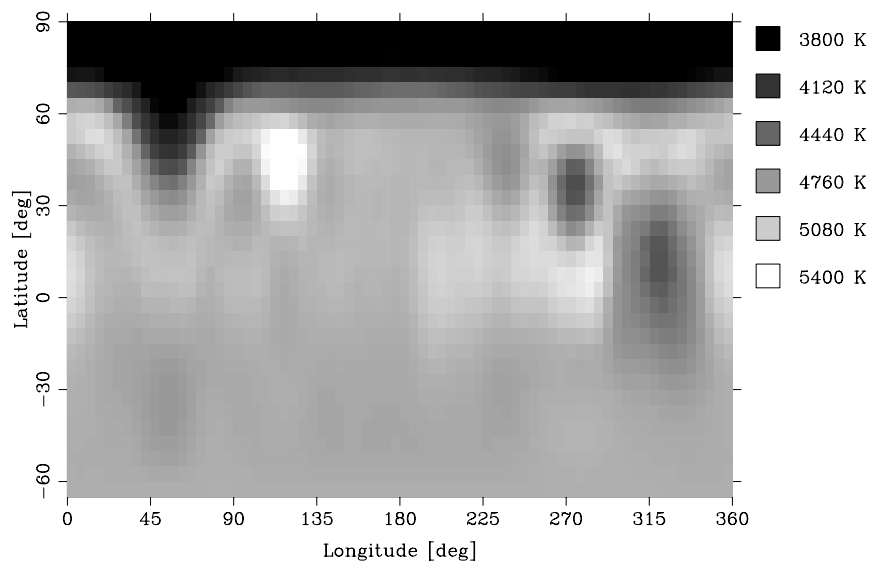

\section{$\Delta \mathrm{I}=-0.005$, abund.$=$ adjusted}
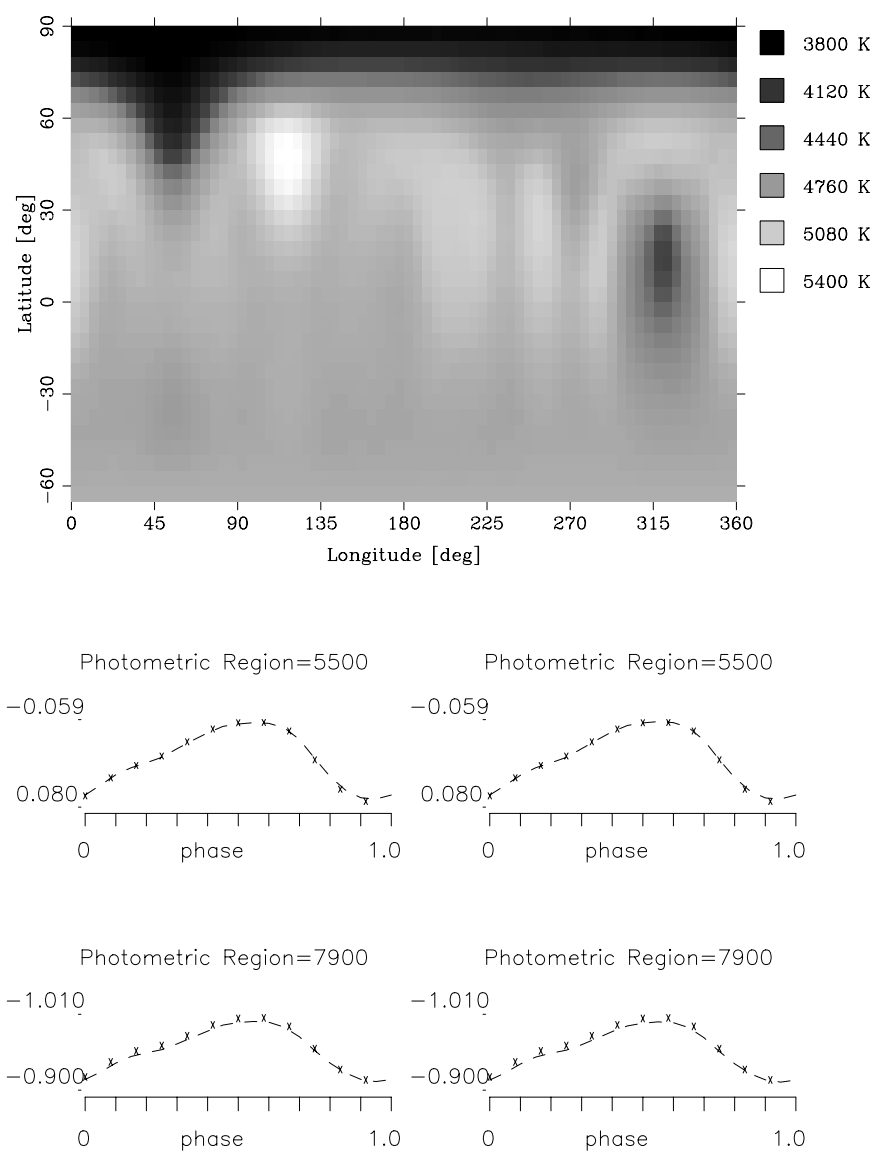

Fig. 5. The effects of a constant continuum displacement, $\Delta I$, for all line profiles. Top images with $\Delta I=+0.005$, bottom images with $\Delta I=-0.005$. Shown is only Case 1 . The left images were recovered without abundance adjustment while the right images were recovered with abundances adjusted by $+0.05 \operatorname{dex}$ (top) and $-0.04 \operatorname{dex}$ (bottom). The lower panels show the $V$ and $I_{\mathrm{c}}$ light curves (crosses) and their respective fits. From left to right: without abundance adjustment and $\Delta I=+0.005$ and $\Delta I=-0.005$, respectively, and with abundance adjustment and again $\Delta I=+0.005$ and $\Delta I=-0.005$. Note the inability to fit the $V-I$ color if the abundance is not adjusted 
inclination of $90^{\circ}$. Figure 1 shows the artificial input map in two commonly used projection styles (Mercator and spherical). The line profiles from the forward calculation are computed for 18 equidistant rotational phases and with 91 equally spaced pixels per profile. The artificial light curve was adopted with 12 equidistant points per bandpass. The artificial light and color curves are not shown in this figure but will appear later in the figures with the test recoveries. Our input map was designed to include the commonly encountered surface structures of active stars like a cool polar spot with an asymmetric appendage, several isolated smaller spots at medium latitude, a two-component circular spot simulating an umbra and penumbra analog at the stellar equator, a hot spot with a temperature of $400 \mathrm{~K}$ above the photospheric temperature, and an equatorial band of very weak temperature contrast on one side of the star.

In the following, we compare recoveries with two sources of errors: first, errors in the (artificial) data and, second, errors in the adopted recovery parameters.

\section{Data errors}

\subsection{Photon noise}

The first test considers image reconstructions from spectra with a large range of signal-to-noise $(S / N)$ ratios. We add random noise to the profiles for both test cases to simulate $S / N$ ratios of $3000,900,600,300,150$, and $75: 1$, and then recover the image using Tikhonov and maximumentropy inversion regularizations. The series of maps in Fig. 2 compare some of the results while Fig. 3 shows the "observations" and the respective fits for both cases and for $S / N=150: 1$. This $S / N$ represents typical observations of a $V=11$ th-magnitude star with a $4 \mathrm{~m}$ class telescope, a spectral resolution of $R=100000$ and a 50-min integration time (e.g. as achieved for the T Tauri star HDE 283572 with the 3.6-m CFHT; Strassmeier \& Rice $1998 \mathrm{~b})$. Note that a case of $S / N=75: 1$ has never been used for real applications so far although Barnes et al. (1998) used spectra with a $S / N$ of as low as 45:1 but boosted this to a $S / N$ of over 1000:1 for a co-added line profile using least-squares deconvolution. The maps in Fig. 4 are difference maps between the original input map and the recovery and emphasize the surface regions of increased sensitivity.

All inversions correctly recover the temperature and size of the polar spot and its appendage. The low-inclination case (Case 2) proves to be more of a challenge though than the high-inclination case (Case 1 ). This is because the low-to-moderate latitude spots, especially the two spots at $\ell=230^{\circ}$, compete for recognition against the polar feature. This causes mild gaps in the otherwise homogeneous polar cap that mimic weak polar appendages. Except maybe for the highest
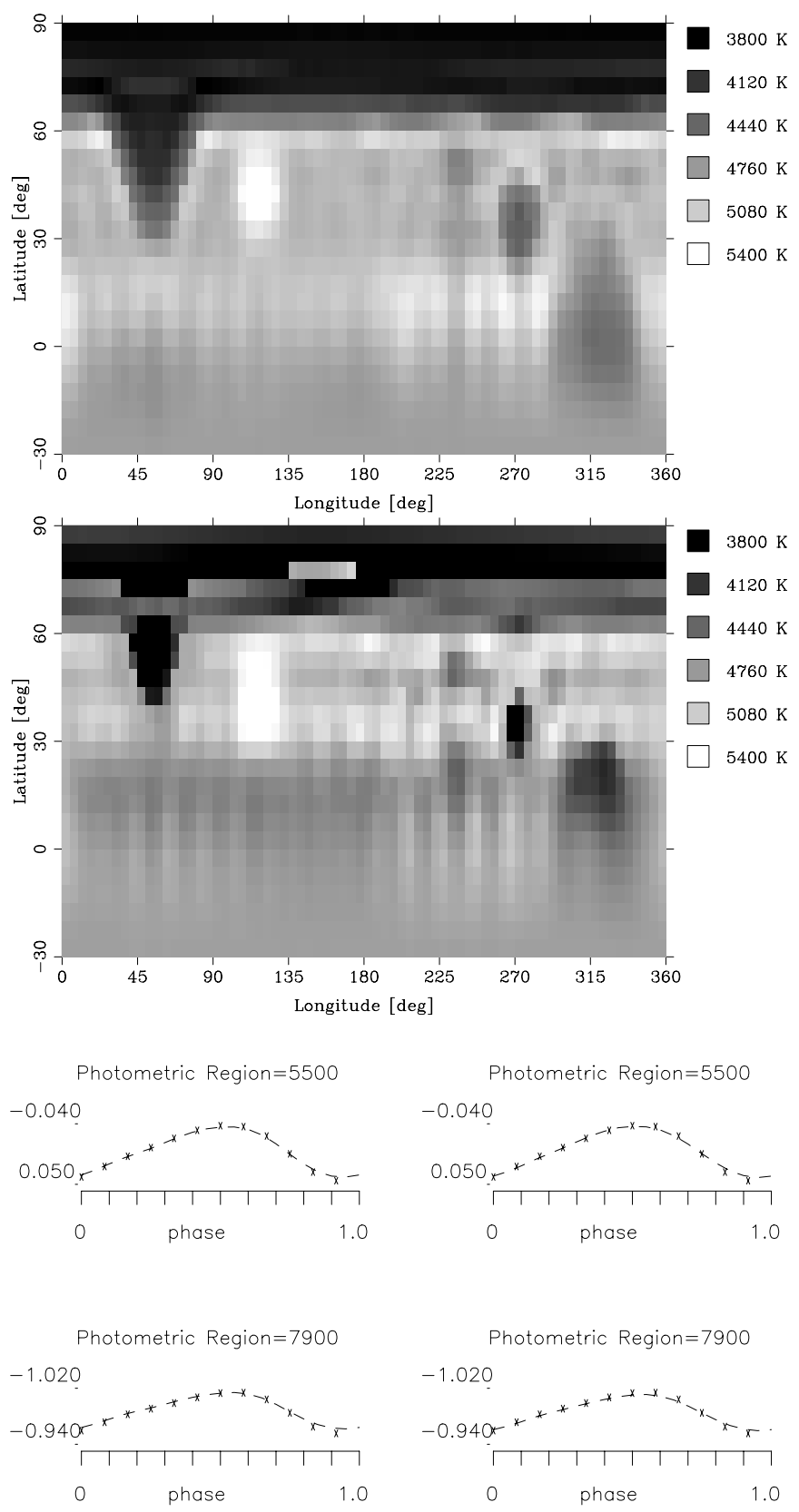

Fig. 6. As in Fig. 5 but for Case 2 (only the abundance adjusted version is shown). The two images were recovered with abundances adjusted by +0.04 dex (top) and -0.06 dex, respectively

$S / N$-ratio spectra of Case 2, the double spot is never resolved. None of the reconstructions reproduced the cool umbra of the large equatorial spot at $\ell=320^{\circ}$ nor the weak equatorial band between longitudes of $10-190^{\circ}$. On the other hand, all reconstructions sucessfully recovered the small hot spot at $\ell=120^{\circ}$ as well as the single, intermediate latitude feature at $\ell=270^{\circ}$. Overall, we conclude that $S / N$ ratio alone does not significantly 
improve the recovery once $S / N \approx 300: 1$ is surpassed if all other factors are perfect.

\subsection{Constant continuum displacement}

Spectroscopists define a continuum as the fit to the upper envelope of an absorption-line spectrum. The various astronomical data reduction packages offer many ways to achieve this fit, e.g. either a low-order polynomial or a bicubic spline and sigma-clipping might be used in IRAF, the NOAO image reduction and analysis facility (available at iraf.noao.edu). Fitting two-dimensional echelle spectra across many orders is usually more complex than a singleorder coudé spectrum and systematic errors may creep into particular spectral regions. This is usually negligible for good $S / N$ spectra of relatively normal stars but may amount to up to $\pm 1 \%$ for very cool stars with broad absorption lines and many blends.

In this and the following chapter, we test the effects of a systematic misfit of the continuum during the data reduction process. First, we recover our two test cases with a continuum offset, $\Delta I$, of $+0.5 \%$ and $-0.5 \%$, respectively (Fig. 5, left two images). A positive $\Delta I$ means a higher continuum and therefore the line depth is artificially increased. The effect on the recovered maps is very pronounced because the missing, or additional, line flux must be distributed across the stellar disk without adding or removing spots that are not in the data. It usually makes the average temperature lower by $\approx 200 \mathrm{~K}$ when the continuum is too high by 0.005 , and $300 \mathrm{~K}$ warmer when the continuum is too low by 0.005 . The broad-band $V-I$ color can not be fitted in any of the two cases while the fit to the line profiles is of comparable $\chi^{2}$. Then, we iteratively adjust the abundance so that it still matches the equivalent width of the spectral line but also fits the light and color-curve zero point (Fig. 5, right two images). This is what we usually do once the continuum offset is fixed (or went unnoticed). This allows a reasonably correct recovery of the average temperature $( \pm 50 \mathrm{~K})$ and the individual feature's temperature contrast but still creates several artifacts, especially noticable at high latitudes in the lowinclination case (i.e. Case 2 in Fig. 6). However, errors in the adopted abundances are related to the adopted atomic line parameters, to the amount of synthesized blends, and to the uncertainty of the rotational velocity and are usually of the order of $10-50 \%$ (depending on spectral type and luminosity, pre-main sequence or post-main sequence, etc.).

\subsection{Continuum slope}

Another type of continuum error appears if the continuum is overfitted, e.g. when a polynomial with too high a degree was used for setting the continuum. For a particular narrow wavelength region of only a few Ångströms, the continuum subtraction could thereby introduce a local slope of the spectrum and the shape of the line profile becomes slightly asymmetric (this is a small effect and usually unnoticable by visual inspection of the spectrum). It appears significant only for the extreme cases of very cool and rapidly rotating stars, e.g. for the pre-main sequence star V410 Tau (K4) or for the double-lined eclipsing binary YY Gem (dMe+dMe), where molecular TiObands contribute to obscure the true continuum.

Figure 7 compares the impact on the two testcases and also shows the actual input data and the fits. The $S / N$ ratio was chosen as high as possible in order to make the effect clearly visible. Compared to "real" data of limited $S / N$ the test case is clearly exaggerated. The right panels in Fig. 7 show a recovery for Case 2, where we expect the larger effect due to the larger full width of the spectral line at continuum level. The input data were artificially changed to mimic a continuum slope of $-1 \%$ to $+1 \%$ from the blue side of the line profile to the red side, i.e. a total of $2 \%$ throughout the full width at continuum level. We find all features correctly recovered, even the small hot spot, but the average temperature of the polar spot appeared lowered by $150 \mathrm{~K}$ compared to the corresponding recovery without a continuum slope in Fig. 2. Case 1 did not have this problem. If the same test is made with $S / N \leq 150$, the polar brightening effect is buried in the noise.

\subsection{Scattered light in the spectrograph}

Scattered light in our artificial data is created by adding in $4 \%$ of white light before the recovery in order to simulate the most common form of scattered light. The recovery is then made with the initial stellar parameters, just as if the scattered light went unnoticed in the data reduction.

Figure 8 compares the reconstructed maps for both cases. A Tikhonov regularisation with a low smoothing factor was adopted and the $S / N$ of the artificial data was 3000:1. A small change of about 0.03 in the logarithm of the abundance was adopted to allow for the small change in the line strength. All main features are correctly recovered but we find that the polar region of the low-inclination case (Case 2) is more prone to artifacts than in the high-inclination case (Case 1). On the other hand, high-to-moderate latitudes and the equatorial regions are more affected in Case 1 than in Case 2. Bright bands with $\Delta T \approx 100 \mathrm{~K}$ above photospheric or, for Case 2, a polar brightening are the results. The temperature of individual features can increase with respect to the input image by up to $700 \mathrm{~K}$. The largest increase is seen, as expected, for the two smallest spots at $\ell \approx 225^{\circ}$ that are barely recovered. This behaviour is due to the effective change in the observed profile when scattered light remains uncorrected. Note that the behaviour in Case 1 is quite similar to what we see when the macroturbulence is modified substantially as in Fig. 12a. It is interesting that when more modest amounts of scattered light as well as 


\section{Continuum slope :}
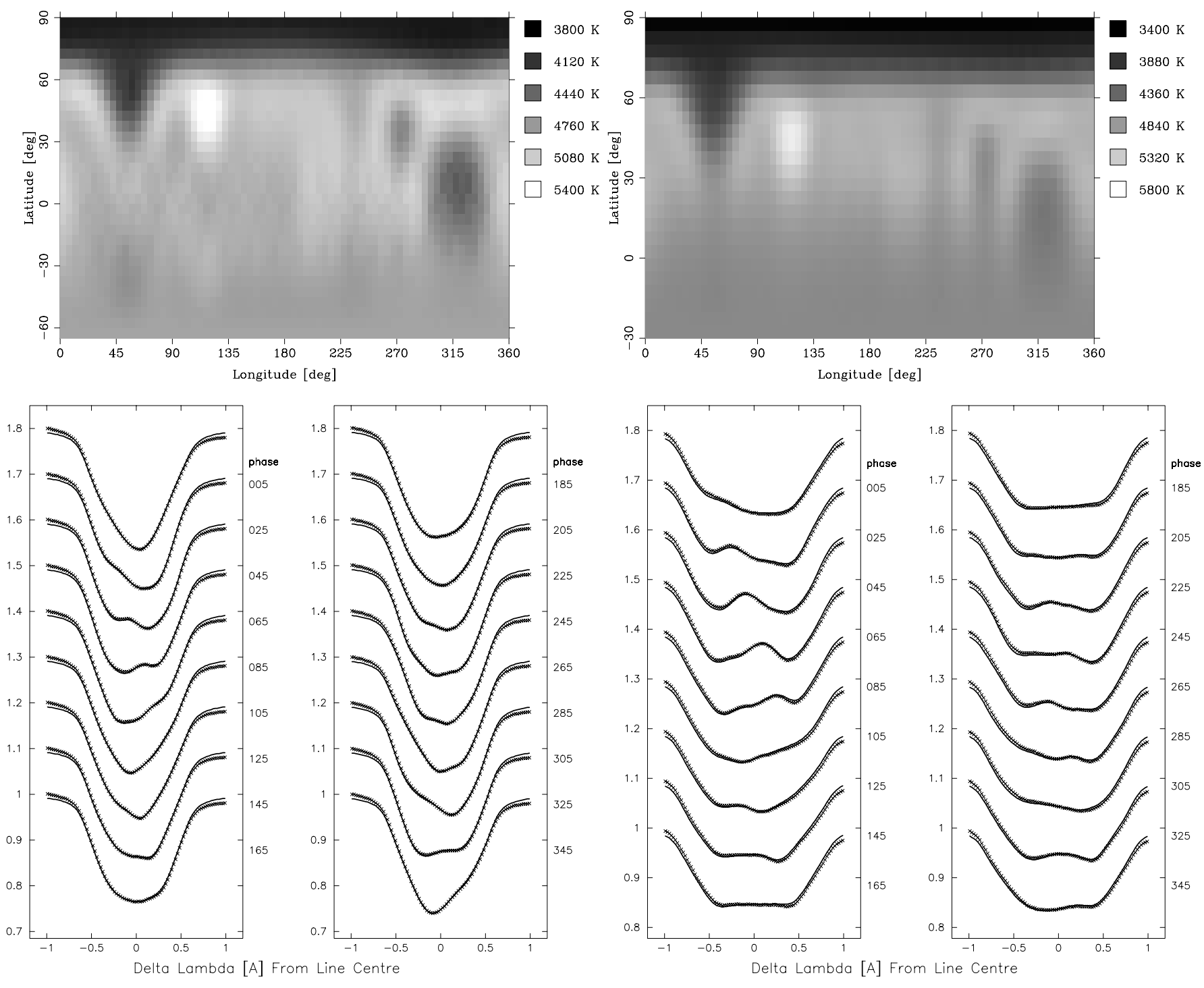

Fig. 7. The effects of a continuum slope of $\pm 1 \%$ for Case 1 (top left) and Case 2 (top right). $S / N$ of the artificial data was chosen as high as possible (3000:1) in order to isolate the slope effect (bottom panels, respectively)

macroturbulent errors are all introduced together as in our final test case with realistic amounts of error for many parameters all introduced at once, the banding effect is far less pronounced.

\subsection{Phase gaps}

All previous recoveries in this paper were made with optimal phase coverage, we used 18 line profiles equidistantly distributed. This is only seldomly achieved with real observations given interruptions due to bad weather, telescopetime limitations etc. In this section, we will successively remove more and more line profiles from the input and investigate the increasing detoriation of the recovered images. However, all maps were obtained with full phase coverage for the photometry.

In the first test only two successive phases are removed, according to a phase gap of 0.167 or $60^{\circ}$ in stellar longitude. Then, we remove three successive phases (a gap of 0.222 or $80^{\circ}$ ) and four successive phases (a gap of 0.278 or $100^{\circ}$ ). Figure 9 compares the obtainable image quality for input data with $S / N=300: 1$ and no external errors. In such a situation the recoveries are better than intuition would suggest, even directly at the phases that are not covered by spectroscopic observations. Note that TEMPMAP shifts more weight from the profile data to the photometric data when a (to be specified) gap occurs. In the present case this was only relevant for the $100^{\circ}$-gap tests. 
$4 \%$ scattered light :
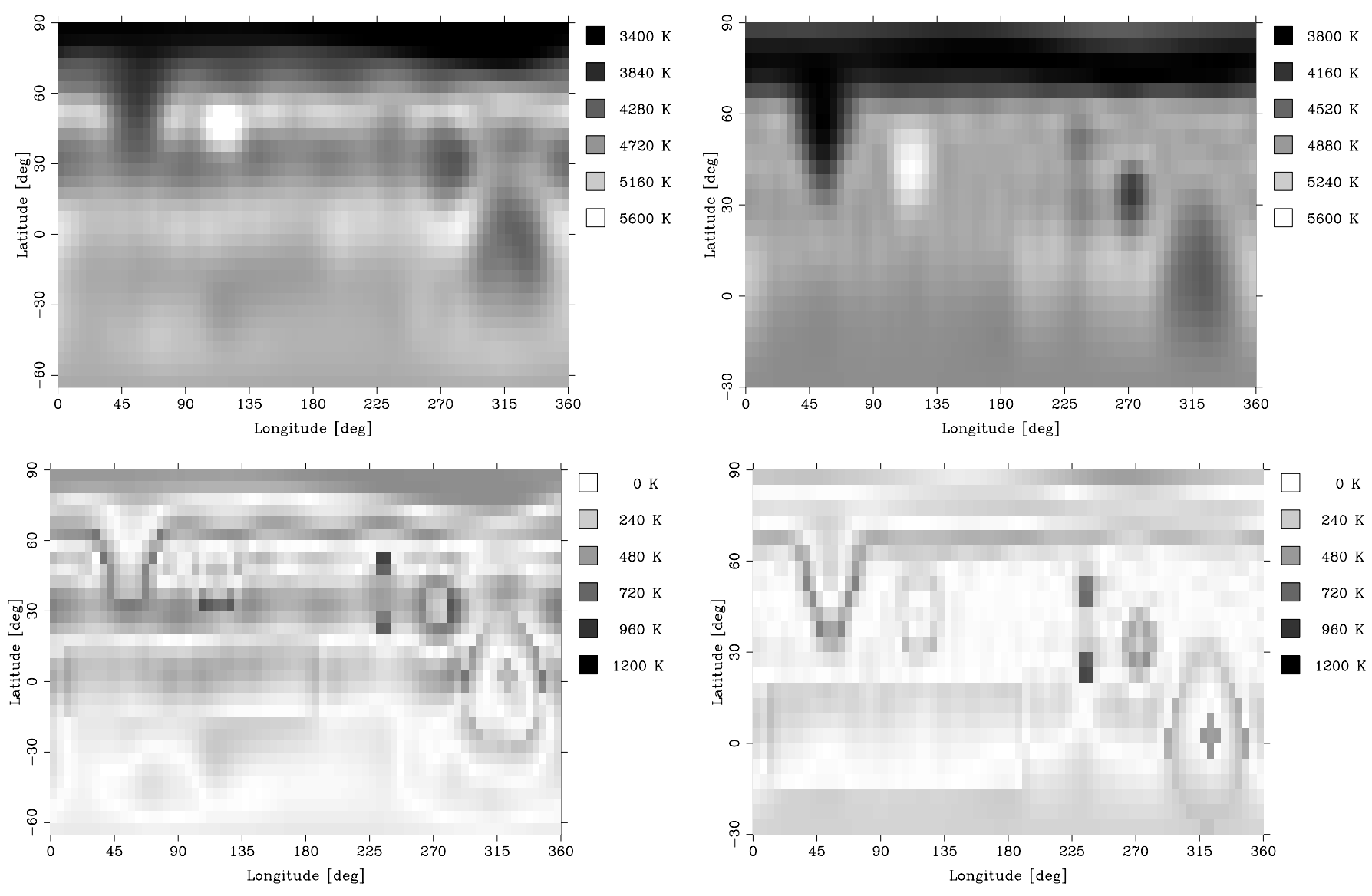

Fig. 8. The effect of unnoticed scattered light in the spectrograph. Left column: Recovery of Case 1, right column: Case 2. The lower panels are the difference maps in the sense input minus recovery. The total amount of scattered light was $4 \%$, which is a common number for cross-dispersed echelle spectrographs. The two top images should also be compared with the input map in Fig. 1. Notice the artificial polar brightening for Case 2 and the artificial bright and dark bands at low and intermediate latitudes for Case 1

\subsection{Phase gaps without photometry}

The same series of inversions from the previous section is now made without any photometry as input. For real applications, we shift more weight to the continuum once a preset phase gap of approximately $40-50^{\circ}$ in the spectrum coverage occurs. The recovery of the missed regions on the star is then dominated by the light and color curve information rather than the spectral line profile. A worst case scenario in real observations occurs when we are left with unevenly distributed spectra with large phase gaps and no photometry. The result of such a test is shown in Fig. 10.

We find that the recovery is essentially independent of the $S / N$ ratio of the data once $S / N$ is greater than $\approx 300: 1$. Even a phase gap of as large as $100^{\circ}$ does not significantly affect the recovered features. Of course, this test assumes randomly distributed photon noise and that no external errors are present; a situation probably never achieved with real data. However, the test clearly demonstrates the robustness of our code.

\section{Input-parameter errors}

\subsection{Confusion in recovery of hot vs. cool spots}

For this test, we moved the single hot spot in our original input map (Fig. 1) to different latitudes to see whether we can still recover its correct location and whether it influences the recovery of the cool spots. Two tests are made, one with the hot spot close to the stellar equator spanning the latitude range $0^{\circ}$ to $25^{\circ}$, and the other with the hot spot close to the rotation pole spanning latitudes from $60^{\circ}$ to $85^{\circ}$. Figure 11 shows the results. The lower part of the figure shows slightly higher temperatures than the $5000 \mathrm{~K}$ original temperature of the model because the initial trial temperature for TEMPMAP was set at $5200 \mathrm{~K}$ 
a. Phase gap $=60^{\circ}$ :
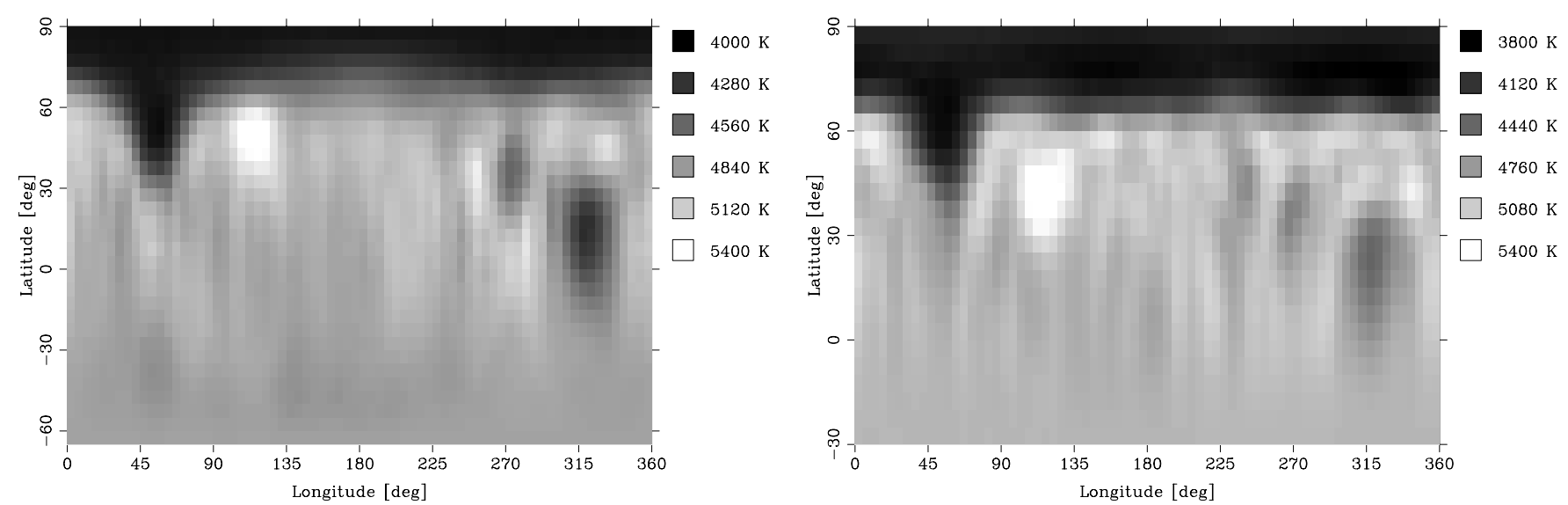

b. Phase gap $=80^{\circ}$ :
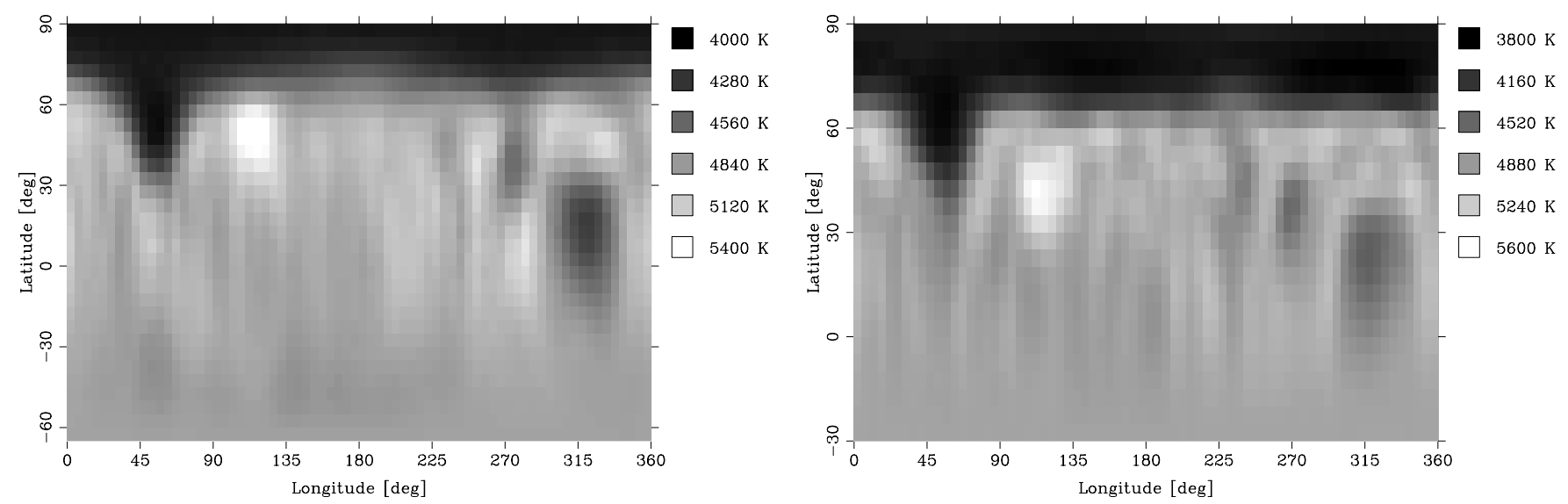

\section{c. Phase gap $=100^{\circ}$ :}
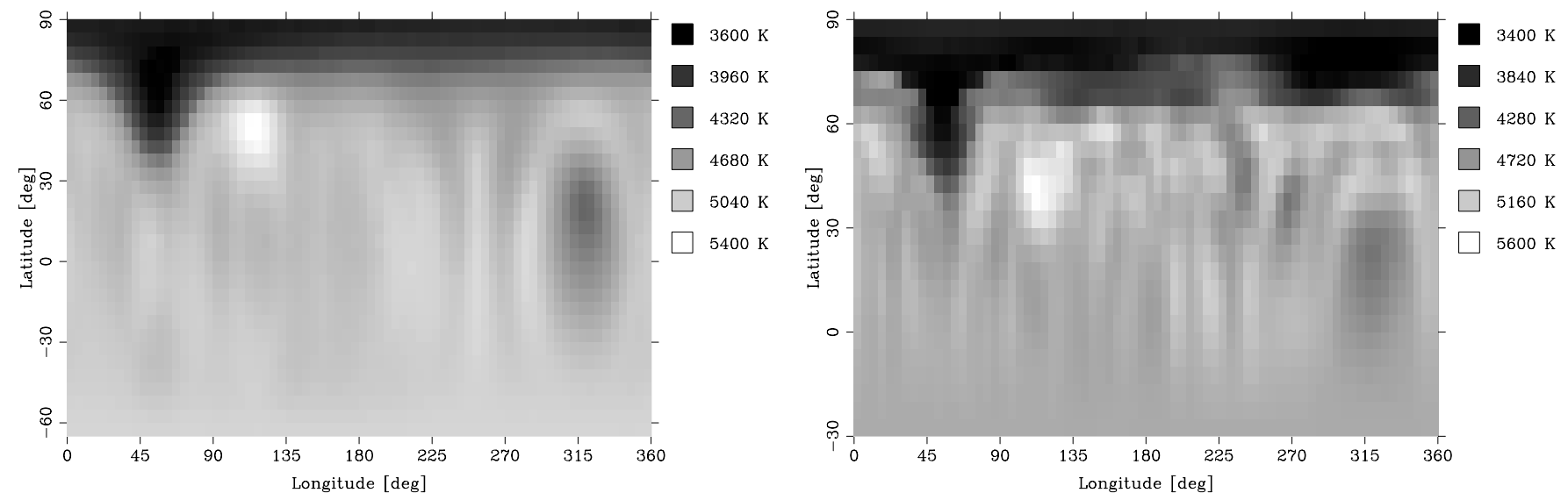

Fig. 9. Recoveries with a spectroscopic phase gap but with continuous photometric coverage. The input spectra have $S / N=300: 1$. Maps in the left column are for Case 1, in the right column for Case 2. From top to bottom: phase gaps of $60^{\circ}(0.167)$ from $305^{\circ}$ to $5^{\circ}, 80^{\circ}\left(0^{\text {p }} 222\right)$ from $285^{\circ}$ to $5^{\circ}$, and $100^{\circ}(0.278)$ from $265^{\circ}$ to $5^{\circ}$ 


\section{a. Phase gap $100^{\circ} ; \mathrm{S} / \mathrm{N}=3000: 1$}

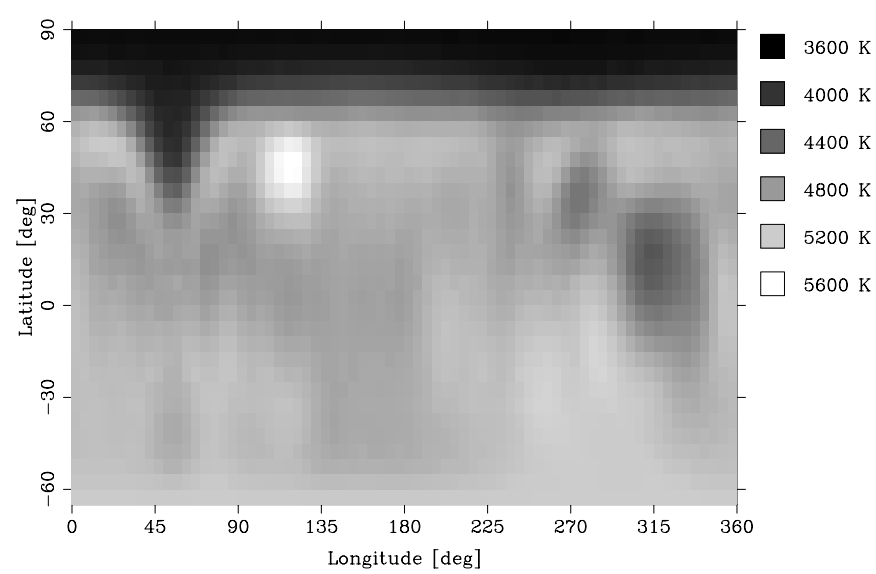

b. Phase gap $100^{\circ} ; \mathrm{S} / \mathrm{N}=300: 1$

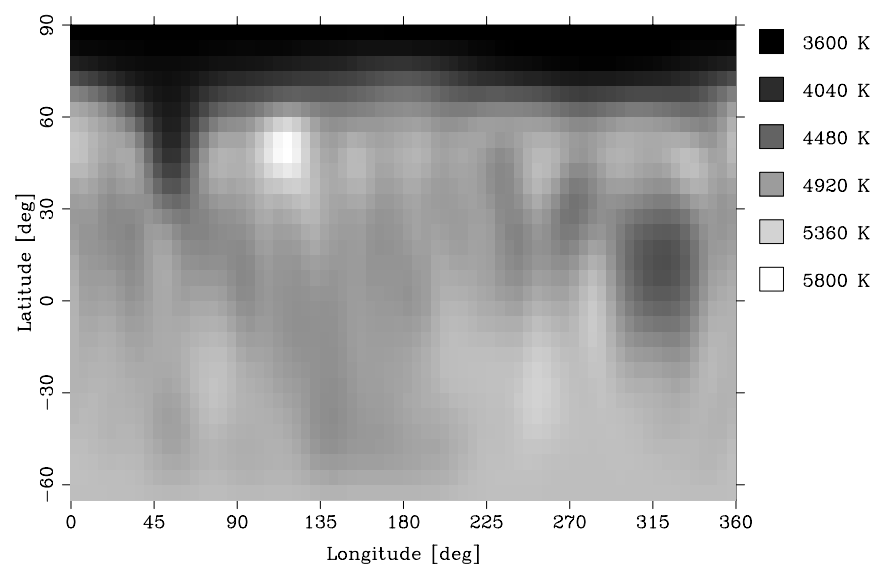

Fig. 10. Two recoveries with a large phase gap and without photometric data: a) for high- $S / N$ input spectra (3000:1), b) for realistic $S / N$ spectra (300:1). Only Case 1 is shown. A correct recovery was possible for both cases even within the phase gap between $\ell=265^{\circ}$ to $5^{\circ}$

to simulate more realistic situations where the temperature of the unspotted photosphere is unknown. In those "southern", rarely observed parts of the star the program has little incentive to modify the temperature from the starting value and limited regions with slightly elevated temperature can be seen. No area in the originally unspotted part of the star was recovered at a temperature of more than $50 \mathrm{~K}$ above the recovered temperature of the surrounding unspotted regions of the original model except immediately adjacent to very dark regions such as the polar extension where the temperature "rebounds" to about $150 \mathrm{~K}$ above the original (although threshold effects in the figures occasionally exaggerate the differences in adjoining regions). A test with a model completely without a hot spot on the surface showed no recovered temperature above $5100 \mathrm{~K}$ (or $100 \mathrm{~K}$ above the original) anywhere above a latitude of $-40^{\circ}$ in Case 1 except, again, immediately adjacent to the polar extension where the temperature reached $5150 \mathrm{~K}$. We conclude then that Fig. 11 shows an excellent recovery for both cases and this then strengthens our findings of warm spots on the WTTS V410 Tau (Strassmeier et al. 1994; Rice \& Strassmeier 1998) and on the K0 giant XX Tri (Strassmeier 1999). Note though that for Case 2 (the lower inclination case) the high-latitude hot spot affects the temperature distribution within the cool polar cap by up to $1000 \mathrm{~K}$ in the immediate vicinity but not so for Case 1. This is a geometric projection effect because the latitudinal location of the hot spot appears the more elongated towards the back hemisphere of the star the lower the inclination.

\subsection{Atmospheric parameters}

It has been argued (e.g. Byrne 1996) that an active star's atmosphere undergoes large amounts of non-radiative heating which may influence their upper photospheres. This, in turn, could give rise to a filling-in of the line cores of strong photospheric lines. Recent non-LTE synthesis of the Ca I $6439-\AA \AA$ line (the most commonly used spectral line) showed that its flat-bottomed profile structure can not be synthesized with chromospheric activity at the visible rotation pole and that the results are practically identical for strong and weak lines and thus agree with the standard case of a LTE assumption (Bruls et al. 1998). A direct comparison of Doppler images of AB Dor from the strong sodium D lines with several weaker photospheric lines also did not show an artificial polar spot (Unruh \& Collier Cameron 1997). Nevertheless, we agree with the arguments of Byrne (1996) above and perform tests in which we adopt certain atmospheric input parameters for the forward problem but then invert the artificial data with wrongly chosen atmospheric parameters.

\subsubsection{Radial-tangential macroturbulence}

The first test deals with macroturbulence and its radial and tangential isotropy across the stellar disk. As is usual in applications to solar-type stars (e.g. Gray 1992), we adopt a radial-tangential macroturbulence velocity with equal radial and tangential components as our standard case (listed in Table 1). The forward computations are now done with a purely tangential velocity component of $5 \mathrm{~km} \mathrm{~s}^{-1}$ but the reconstructions are performed with the parameters of the standard case, i.e. $3.5 \mathrm{~km} \mathrm{~s}^{-1}$ and isotropic. In Fig. 12a, we show the maps recovered for Case 1 and Case 2, respectively, together with their difference maps with respect to the input map in Fig. 1. We choose the highest possible $S / N$ ratio for the artificial data in order to clearly isolate the effect.

Figure 12b is similar to Fig. 12a but that the forward model is computed with a purely radial macroturbulence 


\section{a. Equatorial hot spot:}
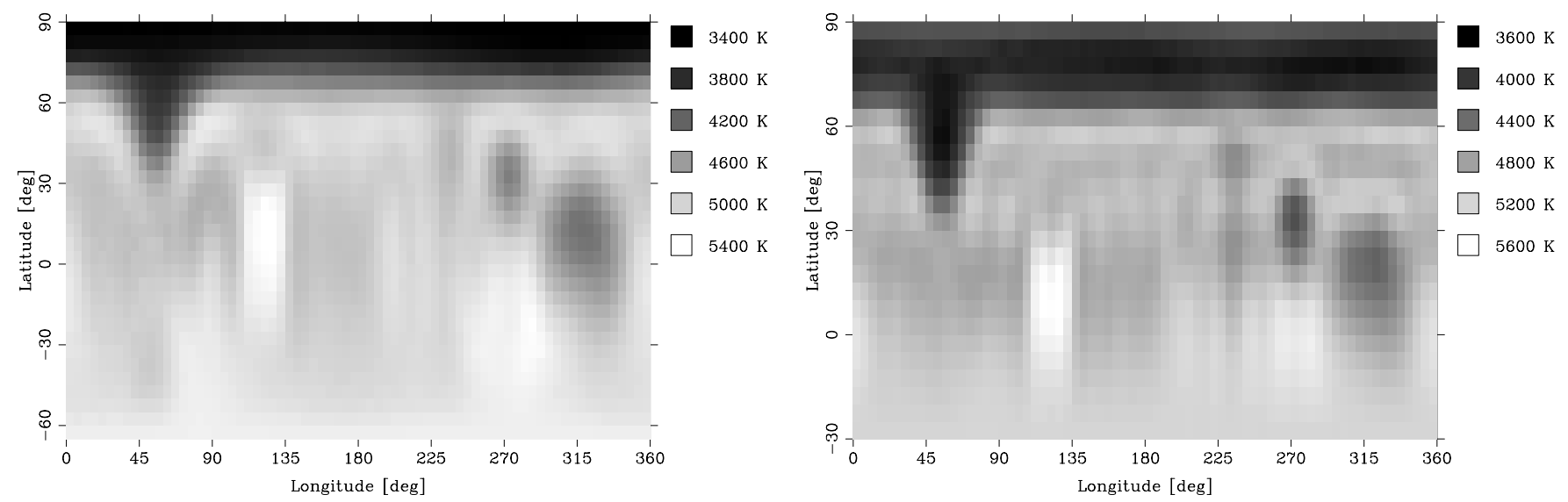

b. Polar hot spot:
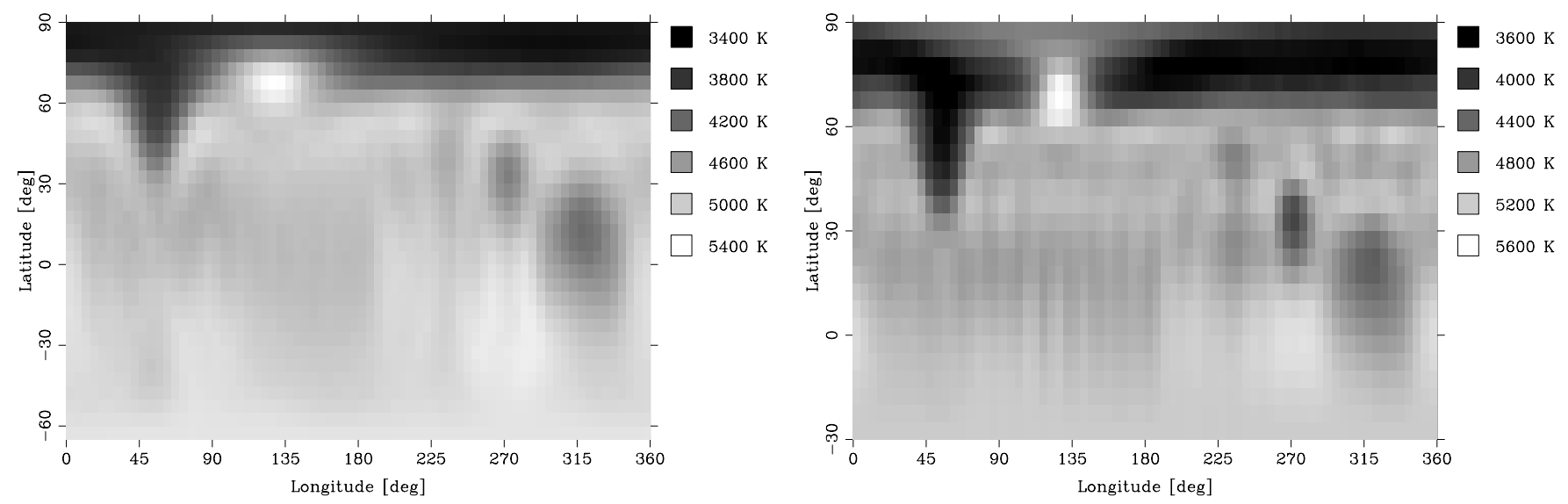

Fig. 11. Recovered maps with the original hot spot placed on a) the equator and b) close to the pole. A correct recovery is possible, even for hot spots. Left panels are for Case 1, right is for Case 2. Note the local brightening of the polar spot in the vicinity of the hot spot (panel b), right map)

model and then recovered with the standard-case parameters. Both figures thus represent the most extreme influence possible and cause the generation of a dark band at intermediate latitude for Case 1. A test with more moderate values for the anisotropy and also for smaller differences of the amount of macroturbulence resulted in maps so similar to the originals that we do not show them separately.

\subsubsection{Gravity}

For the radiative transfer calculation within TEMPMAP, we usually input up to 10 model atmospheres of different temperature but, of course, the same gravity. Our goal now is to estimate the influence of a set of atmospheres with wrongly chosen $\log g$. The value for $\log g$ is usually pre-estimated from a trial-and-error fit of a synthesized spectrum to one or several particularly gravity-sensitive lines like e.g. Ca I 6439 A. Its uncertainty usually amounts to \pm 0.5 dex for giants and \pm 0.25 for G- and early-K dwarfs (see, e.g. Strassmeier \& Rice 1998a).

Figure 13 shows the two maps and light curves for Case 2 reconstructed with wrong $\log g$ atmospheres. Figure $13 \mathrm{a}$ is a test with ten input atmospheres with $\log g=4.5$ (instead of 4.0 as in the forward computation), and Fig. 13b with $\log g=3.5$. Both recoveries have no problem of finding the correct spot geometry, including the polar spot as well as the smaller low-latitude spots. The misfit to the light curve zeropoints (in our case the $V-I_{\text {c }}$ color), however, shows up immediately. The higher equivalent width of the local line profile for the $\log g=4.5$ atmospheres additionally results in a bright band with $\Delta T \approx 240 \mathrm{~K}$ encircling the star between a latitude of $30^{\circ}-60^{\circ}$, while the correspondingly lower value for the $\log g=3.5$ atmosphere results in an overall cooler surface by $\Delta T \approx 200 \mathrm{~K}$. If the gravity mismatch would go unnoticed in a real application, we would alter the abundances of that particular element to increase or 


\section{a. Tangential anisotropy:}
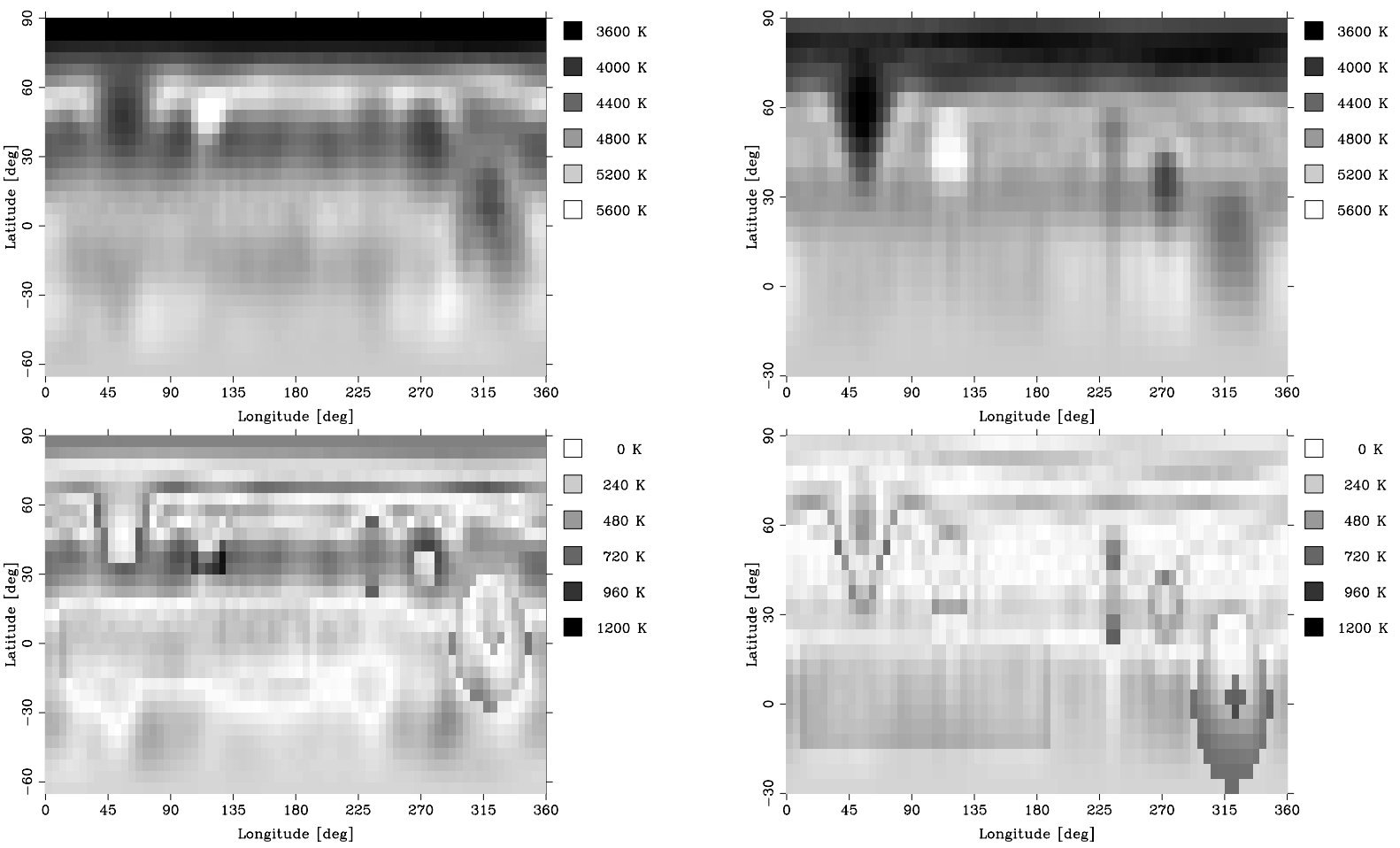

\section{b. Radial anisotropy:}
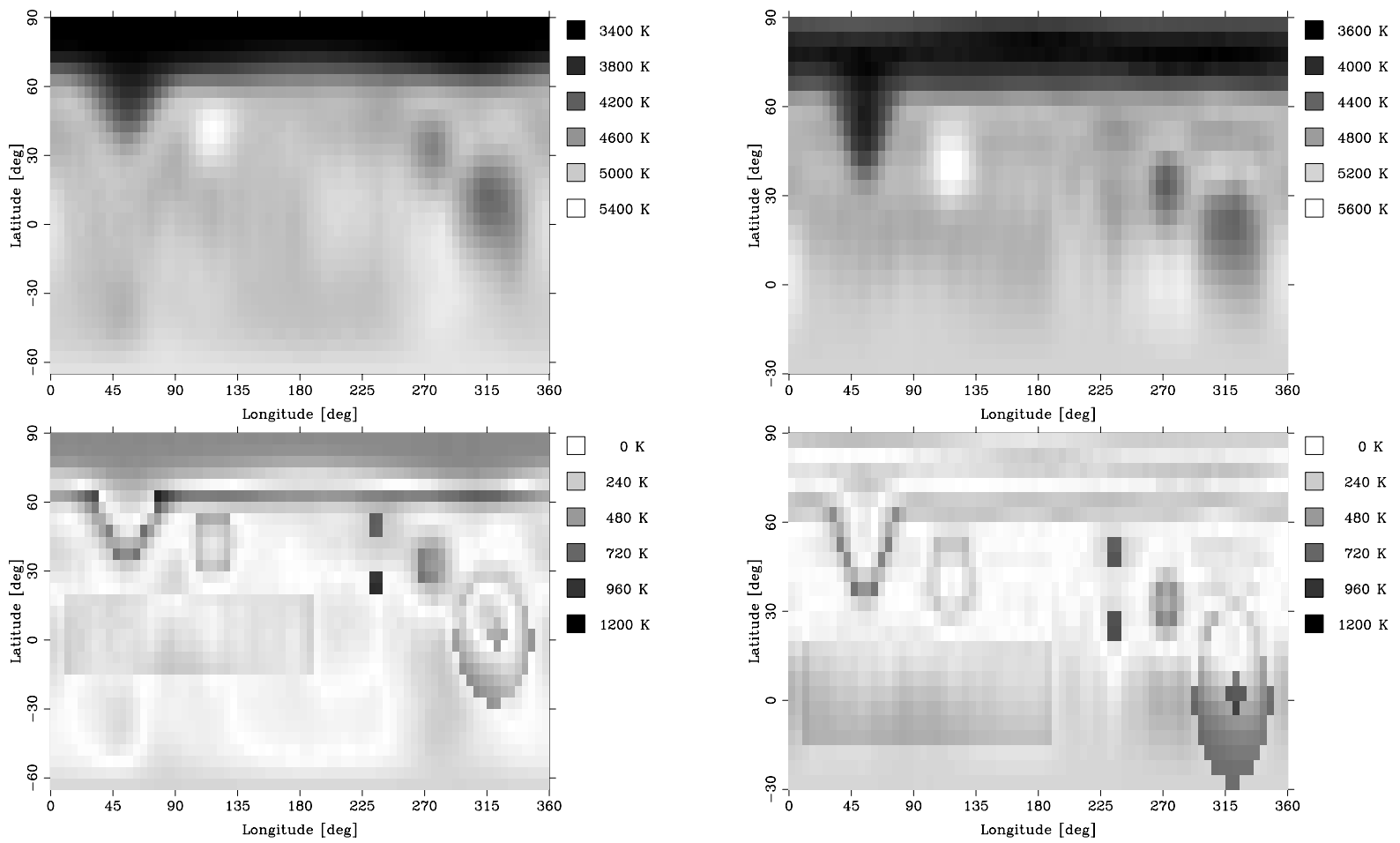

Fig. 12. Effects of the anisotropy of macroturbulence velocity. Panel a) shows the case of a purely tangential macroturbulence but recovered under the assumption of an isotropic radial-tangential distribution. Panel $\mathbf{b}$ ) is the recovery from a purely radial macroturbulence distribution but otherwise identical assumptions. See text 


\section{Gravity:}

\section{a. $\log g=4.5$}

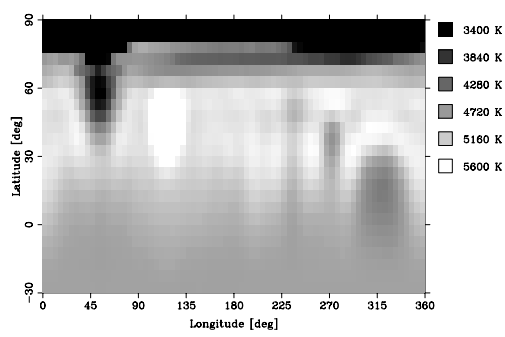

b. $\log g=3.5$

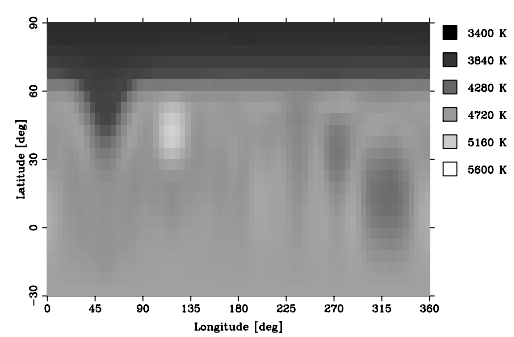

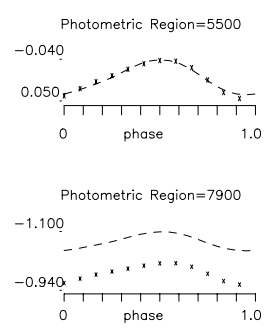

Fig. 13. Tests with model atmospheres of different gravity. a) The input gravity was set to $\log g=4.5$ instead of 4.0 as in the forward computation. b) Here it was assumed $\log g=3.5$. Notice the inability to fit the color-curve zeropoint. Only Case 2 is shown

\section{Sum of the damping constants:}

a. $+0.15 \mathrm{dex}$

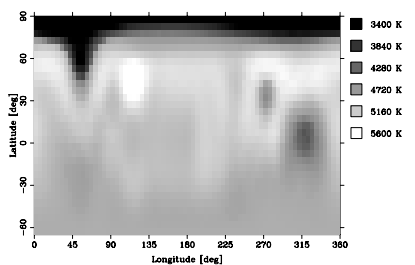

Photometric Region $=5500$

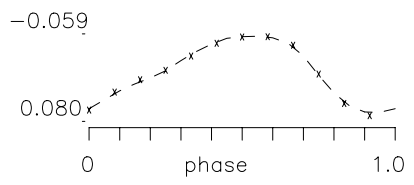

Photometric Region $=7900$

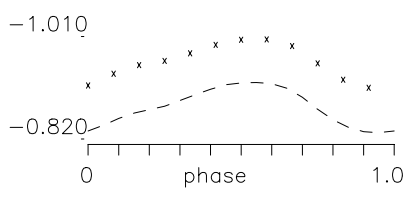

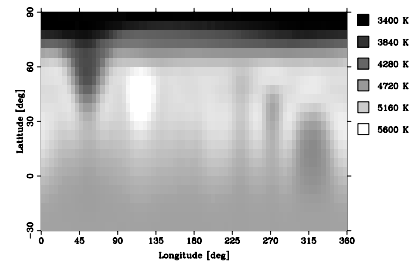

Photometric Region $=5500$

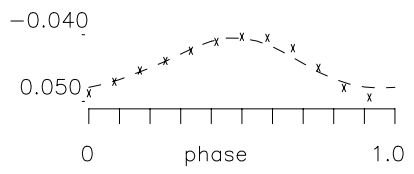

Photometric Region $=7900$

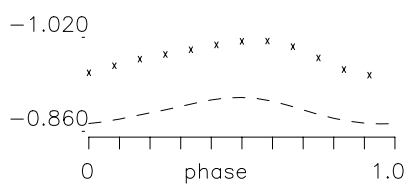

b. -0.15 dex
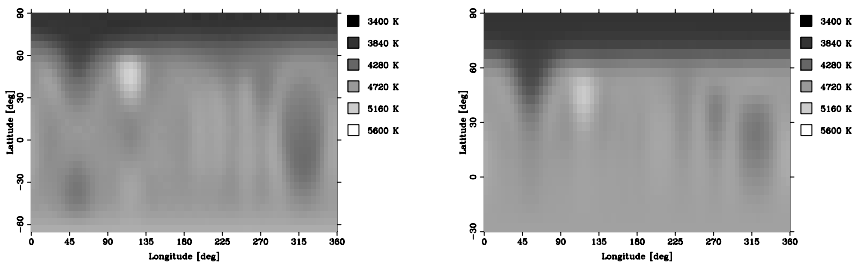

Photometric Region $=5500$

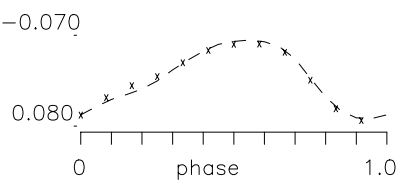

Photometric Region $=5500$
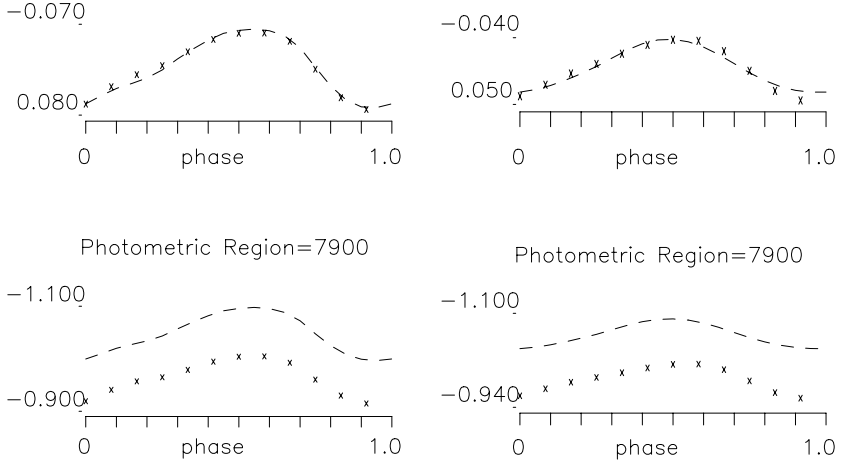

Photometric Region $=7900$

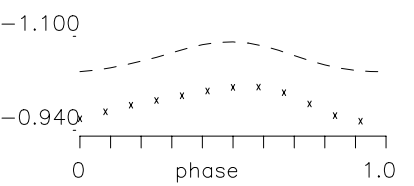

Fig. 14. A test with different line-damping constants. a) A change of +0.15 dex of the nominal value of all three damping constants, b) a change of -0.15 dex. The left column is always for Case 1 and the right column for Case 2 . For each test, we show the reconstructed map and the $V$ and $I_{\mathrm{C}}$ lightcurves and their fits. Note the increasing misfit when all three damping coefficients are altered. A change of \pm 0.15 dex is also the range of values where our code can react in terms of lowering and raising the overall surface temperature. Any change above this value results in a significant misfit of the line equivalent width and spurious hot and cool spots

decrease the equivalent width by an amount that removes such artifacts and agrees with the color zeropoint.

\subsection{Atomic line parameters}

Many atomic parameters of a particular transition contribute to a stellar spectrum. Most notably the central wavelength, the excitation potential, i.e. its sensitivity to temperature, the damping constants, i.e. the population level and its lifetime, and the transition probability, i.e. the strength of a line. In the past, we found that Doppler imaging is mostly prone to wrong values for the transition probability and, to a lesser degree though, to the various damping constants. We will thus concentrate our tests on these two parameters. 


\section{Inclination:}

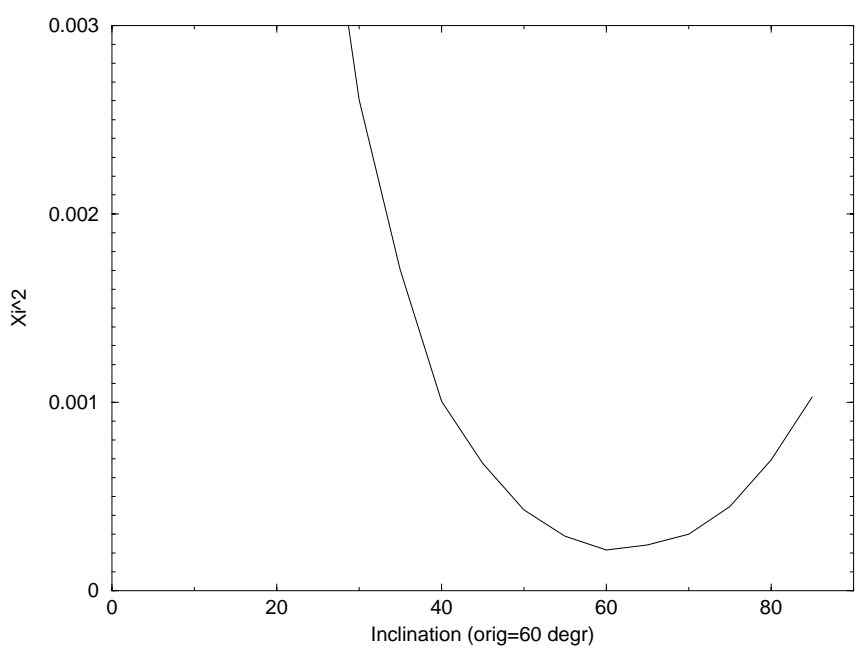

Fig. 15. Reconstruction of Case 1 with inclination angles between $5^{\circ}$ and $85^{\circ}$. The vertical axis is $\chi^{2}$ which here is just the sum of the squared difference between the model profile points and the 91 artificial profile data points. A clear minimum near the correct inclination is obtained

\subsubsection{Transition probability}

The number of electrons per second that will spontaneously jump from an upper level to a lower level is proportional to the Einstein probability coefficient for spontaneous emission or absorption, to the population of the upper or lower level, and to the energy $h \nu$ that separates the two levels. These quantities are linked to the emission or absorption coefficient and thus to the specific intensity of the line. In practice, a single value, the socalled $\log (g f)$ value, determines the line intensity. It is an input parameter in our Doppler imaging and either taken from atomic-line databases such as VALD (Kupka et al. 1999) and/or obtained by fitting the appropriate parts of the solar spectrum (e.g. Valenti \& Piskunov 1996; Strassmeier et al. 1999). It is an important parameter and was tested earlier in Strassmeier (1996) for Ca I 6439, and we refer to that paper since the conclusions did not change for the line used in the present paper. We note that the effects of a wrong $\log g f$ on the maps are indistinguishable from the effects of wrong elemental abundances.

\subsubsection{Damping constants}

TEMPMAP automatically computes three damping constants; one for natural (radiative) damping, one for Stark damping, and one for van der Waals damping. The user has the choice of automatic computation by using the Unsöld (1955) expressions for $\gamma_{\mathrm{rad}}, C_{4}$, and $C_{6}$ or presets his/her own favorite values in the input file. The automatic computation of the three relevant damping constants for the Fe I $6411.649-\AA$ line that are used for the tests in this paper are (in parenthesis are the Kurucz-(1993) values for comparison purposes) 7.732 (7.905), -5.096 (-5.455), and $-7.670(-7.622)$ for radiative, Stark, and van der Waals broadening, respectively. We found that for the temperatures and densities of active G- and K-stars and their cool spots, the natural and the van der Waals broadening are about equal sources of damping, and we thus alter just the radiative damping constant for some of our tests and the sum of all three for the remaining tests.

Figure 14 shows two sets of tests. One has the sum of the damping constants set to +0.15 dex of the nominal value, and the other has the sum set to -0.15 dex. Both tests are made for the high-inclination case (Case 1, $i=65^{\circ}$ ) and the low-inclination case (Case $2, i=30^{\circ}$ ). A value of $\pm 0.1-0.2$ dex represents the "worst-case" uncertainty of this parameter and was estimated from the spread of damping constants in VALD for several neighbouring lines. $\mathrm{A} \pm 1.0$-dex variation in the radiative damping alone turned out to be the amount of change that is required to recognize some significant changes in the maps.

The tests in Fig. 14 show significantly hotter and cooler reconstructions for higher and lower damping, respectively. Still, the color zero point can not be fitted at all and an offset of approximately $\pm 0{ }^{\mathrm{m}} 1$ in $V-I_{\mathrm{c}}$ for $\mp 0.15$ dex, respectively, remains. This would be easily spotted in real photometric data. Furthermore, the line equivalent width that is added (or removed) in order to fit the observations would be compensated for by adjusting the elemental abundances by hand. This is a regular procedure before the actual line-profile inversion with TEMPMAP.

The tests with \pm 0.15 dex of the radiative damping constant alone show practically identical reconstructions compared with the nominal value except maybe for lower damping for Case 2. There, some structure appears in the polar cap that is not in the input map. The only recognizable misfit for both cases is a slight zero-point offset in $V-I_{\mathrm{c}}$ of $-0{ }^{\mathrm{m}} 01$ (i.e. the reconstructed image got hotter by $30 \mathrm{~K}$ ). By the time the radiative damping constant is increased by $1.0 \mathrm{dex}$, the color offset amounts to a full 0.1 (at +1.5 dex even 0.25 ), thus we would observe a severe observational mismatch. However, it can be compensated by adjusting the elemental abundance by just -0.17 dex. We do not show the really extreme cases with damping increased by more than 1 dex because they are totally unrealistic and usually would be spotted in the data even before the mapping because the reconstructed equivalent width becomes about half the observed. Lowered values for the radiative damping have a slightly less pronounced effect on the maps and almost none on the broad-band colors because the van der Waals broadening keeps up the line-equivalent width. 


\section{All errors included:}

\section{a. Spherical projection}
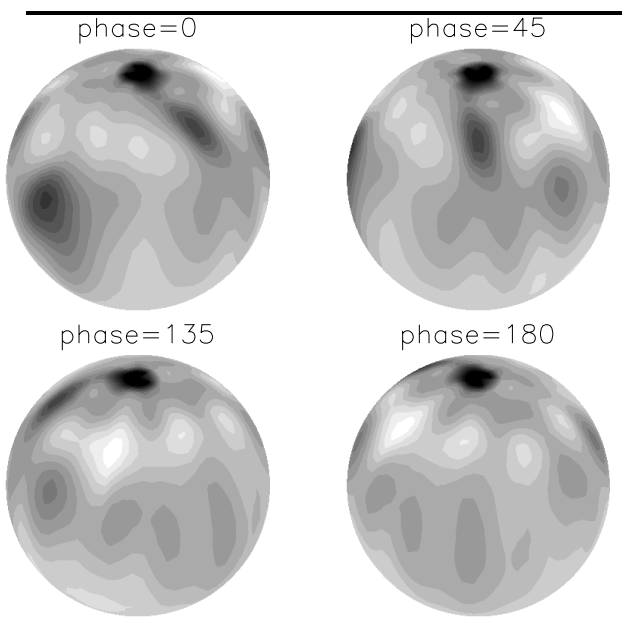

phase $=270$

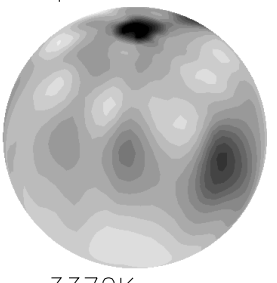

$3370 \mathrm{~K}$

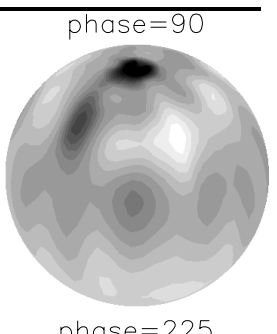

phase $=225$

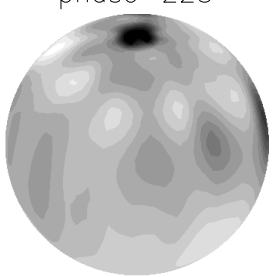

Grid, Phase $=0$

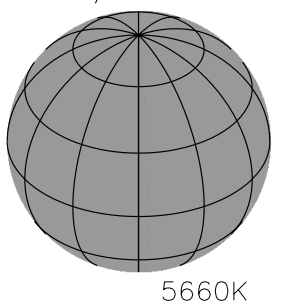

$5660 \mathrm{~K}$

b. Mercator projection

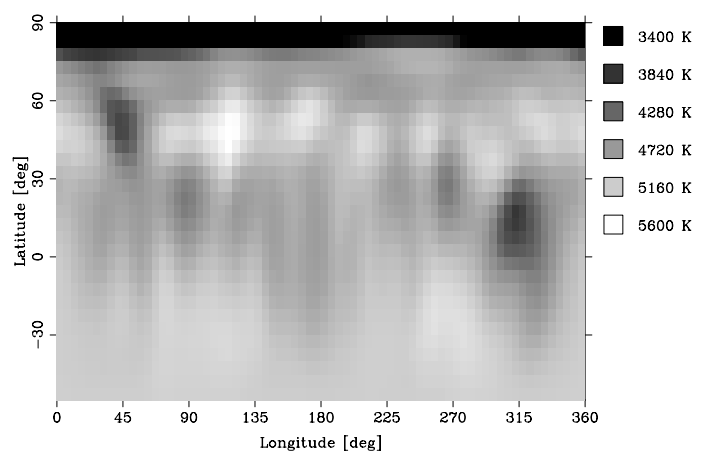

c. Difference map

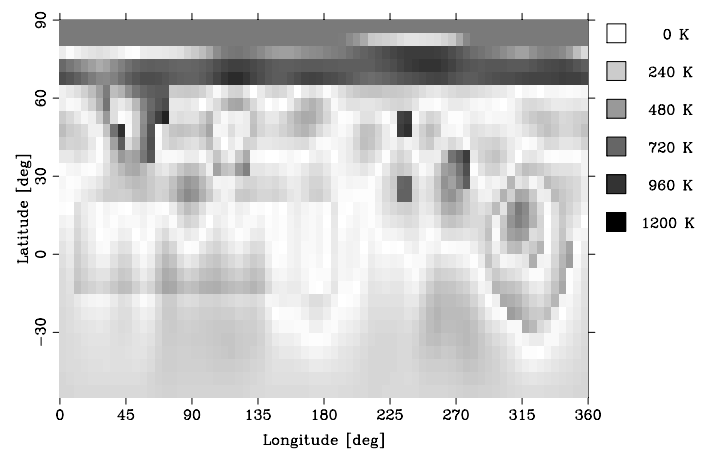

f. Line profiles

\section{d. Light curve}

e. Cross correlation
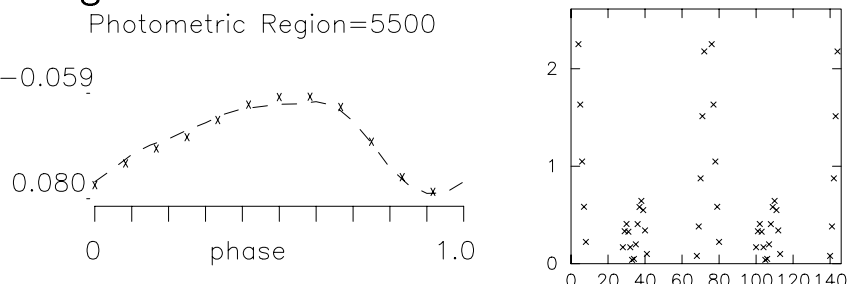

xwidth $=07.57$

Photometric Region $=7900$
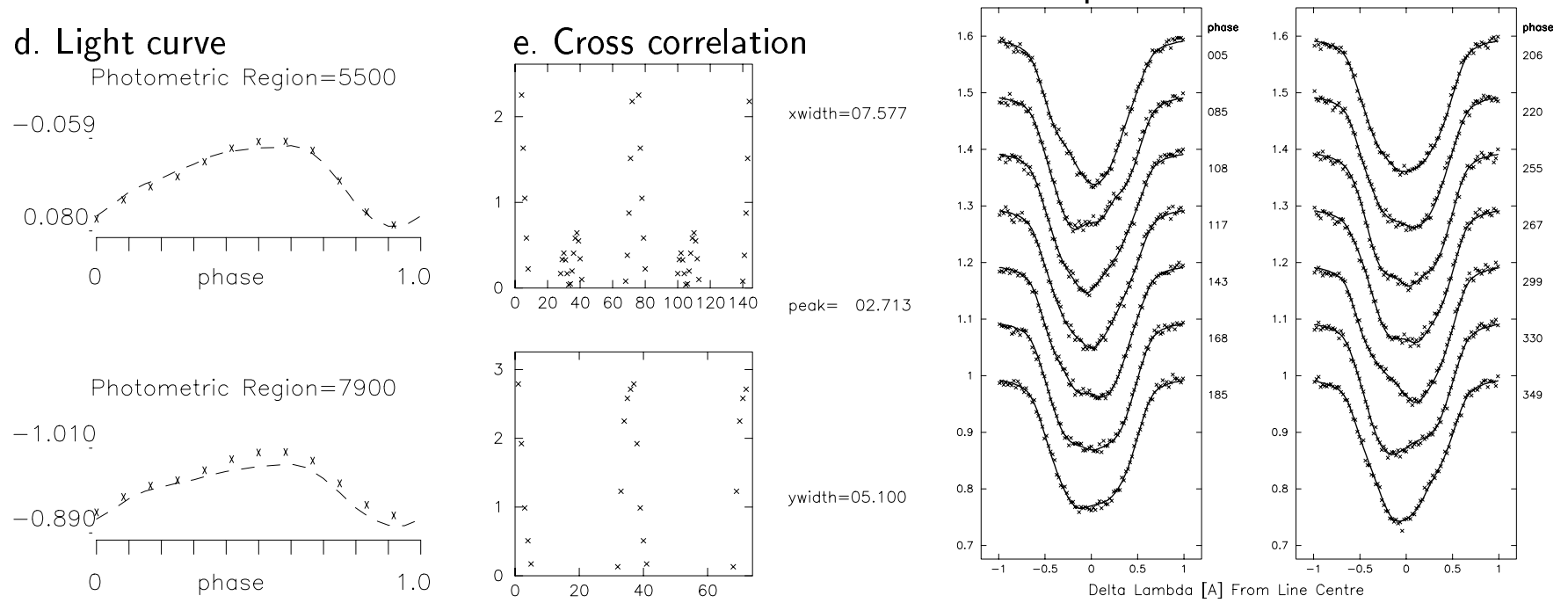

Fig. 16. Test reconstruction with all previous errors included. Case 1 with phase gap between $5^{\circ}-80^{\circ}$. a) Spherical projection, b) Mercator projection, c) difference map between input and reconstructed map in Mercator projection, d) light curve in $V$ and $I_{\mathrm{C}}$ and the fits, e) Cross correlations between input and reconstructed map. Top panel: correlation in longitude given in surface pixels, bottom panel: in latitude (xwidth is the FWHM of the cross-correlation function in longitudinal direction in surface pixels, ywidth in latitudinal direction). The grid is $72 \times 36$ pixels. f) is the artificial line profiles and the fits 


\subsection{Inclination}

In this test, we assume a wrong inclination in the reconstruction and run TEMPMAP on artificial data with $S / N=3000: 1$ in steps of $5^{\circ}$ from $i=5^{\circ}$ to $i=85^{\circ}$. Figure 15 demonstrates that the correct inclination can be recovered just from the run of the $\chi^{2}$ as a function of $i$. The width of the minimum in the curve in Fig. 15 would allow one to adopt inclination values within a few degrees of the nominal value with only a small variation in the goodness of fit. However, there are a lot of other adjustable parameters that need to be optimised at each value of the inclination before a formal error on the inclination can be estimated. Since this was not done in the present test, a $1-\sigma$ error quote is likely too optimistic for real applications but nevertheless illustrates the usefullness of this method of estimating $i$.

In an earlier paper, Hatzes (1996) had shown that lowinclination stars had a more pronounced flat-bottomed shape compared to high-inclination stars. The line-profile shape is thus an indicator for the inclination of the stellar rotation axis and the quality of our fits to real data is such that we can achieve an inclination to within $\pm 10-15^{\circ}$.

\section{5. "Finale furioso"}

In the final test in this paper, we add all previously discussed errors into a single forward computation of the artificial data and then run the image reconstruction on them. The choice of the errors are now such that a reasonable but still worst-case scenario is constructed. Extreme errors, like having the damping constants off by an order of magnitude or more, are not considered. We also proceed as if we had real stellar spectra and photometry and performed a spectrum synthesis to predetermine atmospheric parameters such as $T_{\text {eff }}$ and $\log g$ and then adjust the elemental abundances so that the computed line equivalent width matches the observed one and the photometric zeropoint. The artificial data and its errors applied are in detail:

$-S / N=200: 1$

- Fourteen unevenly distributed profiles;

- A single phase gap of $80^{\circ}$ from $5^{\circ}$ to $85^{\circ}$;

- Continuum slope of $\pm 0.5 \%$;

- Scattered light of $1 \%$;

- Sum of the damping constants off by -0.05 dex;

- Radial macroturbulence is twice as large as the tangential macroturbulence;

- Rotational broadening is overestimated by $0.5 \mathrm{~km} \mathrm{~s}^{-1}$;

- The inclination of the stellar rotation axis is $10^{\circ}$ below the nominal value;

- Fourteen evenly spaced $V I_{\mathrm{c}}$ light-curve points are included in the inversion.

The result for Case 1 is shown in Fig. 16. For the recovery of this image the abundance of Fe was increased from nominal by 0.04 dex. Again notice that the phase gap is now between $5^{\circ}$ and $85^{\circ}$ (instead of $265^{\circ}$ to $5^{\circ}$ ), which clearly affects the reconstruction in this range. First, the polar appendage appears as a separated spot just barely linked to the polar cap and, secondly, an artificial spot appeared at a longitude of $90^{\circ}$ and a latitude of $+25^{\circ}$. Its contrast is very weak though, just $280 \mathrm{~K}$ below the photospheric temperature but probably would have been judged real if reconstructed from real data. We also notice that the polar cap had been recovered with smaller size than in the input map. This causes the steep temperature gradient seen in the difference map (Fig. 16c) at a latitude of $+65^{\circ}-70^{\circ}$. The small double spot at a longitude of $\ell \approx 230^{\circ}$ is missed entirely while the hot spot at $\ell \approx 120^{\circ}$ shows up in the reconstruction. Overall it is evident throughout these tests that while the visibility of features recovered in Doppler images may be under discussion, misplacement of the recovered feature is not a problem. As the reader can see from the figures presented, we can rely on positional information for the assessment of differential rotation or any other secular motion with confidence.

\section{Conclusions}

Doppler imaging of stellar surface structure is a novel technique with similarities to medical brain tomography (instead of a fixed brain and a rotating scanner, astronomers have a fixed spectrograph and a rotating brain, well alright, star of course). The number of free (internal) parameters is at first glance of the order of the number of surface grid points, i.e. $\approx 2600$, and is constrained by the number of input data points (for our test cases 1638 profile points and 24 light-curve points.) This obviously underdetermined situation makes some astronomers uneasy about Doppler imaging but modern inversion algorithms with penalty functions of the form of maximum entropy or Tikhonov etc. together with the surface Doppler-shift constraint from the stellar rotation restricts the freedom of the surface grid points so that we are left with only 11 parameters that are in any sense mathematically free (the eight listed in Table 1 plus three atomic parameters; $\log g f$, damping, and central wavelength). Yet these 11 parameters are still not completely independent or free of physical constraint. For example if $v_{\text {eq }} \sin i$ is measured and $i$ fixed, $v_{\text {eq }}$ is no longer a free parameter. The same is true if we fixed the gravity and the line strength, then the microturbulence is no longer completely free because the equivalent width must be reproduced. This shrinks our problem basically to the common range of uncertainties of stellar astrophysical parameters and atomic line data.

In this paper, we visualized the quantitative influence of the uncertainties of many of the stellar quantities and atomic data. We demonstrated the extreme robustness of our Doppler-imaging code to even exaggerated assumptions, e.g. a recovery with a phase gap of $100^{\circ}\left(0^{\mathrm{p}} 28\right)$ with moderate $S / N$ still correctly recovered the spots located 
within the phase gap. Having simultaneous photometry in two bandpasses not only gives a better handle on the overall temperature of the stellar surface but when used with the line equivalent width the photometry provides a powerful additional constraint so that we are forced to make adjustments in factors such as element abundance to compensate for uncertainty in atomic parameters or $\log g$. This works to minimize the impact on the recovered image when errors in adopted line parameters such as the damping constants occur. We conclude that our stellar maps published so far are relatively free of systematic artifacts and should be considered as the best possible solution for the data available.

Acknowledgements. JBR acknowledges financial support from the Natural Science and Engineering Research Council of Canada (NSERC). KGS is very grateful to the Austrian Science Foundation (FWF) for support under grants S7301AST (APT) and S7302-AST (Doppler imaging). We would like to thank the referee, Dr. Collier-Cameron for a very careful and thoughtful report on this paper. His comments were very useful and supportive.

\section{References}

Barnes J.R., Collier Cameron A., Unruh Y.C., Donati J.-F., Hussain G.A.J., 1998, MNRAS 299, 904

Berdyugina S., 1998, A\&A 338, 97

Berdyugina S., Tuominen I., 1998, A\&A 340, 437

Bruls J.H.M.J., Solanki S.K., Schüssler M., 1998, A\&A 336, 231

Byrne P.B., 1996, in IAU Symp. 176, Stellar Surface Structure, Strassmeier K.G., Linsky J.L. (eds.). Kluwer, Dordrecht, p. 299

Collier Cameron A., 1992, in Surface Inhomogeneities on LateType Stars, Lecture Notes in Physics, Vol. 397, Byrne P.B., D.J. Mullan (eds.). Springer-Verlag, Berlin, p. 33

Donati J.-F., 1999, MNRAS 302, 457

Donati J.-F., Brown S.F., Semel M., Rees D.E., Dempsey R.C., Matthews J.M., Henry G.W., Hall D.S., 1992, A\&A 265, 682

Gray D.F., 1992, The observation and analysis of stellar photospheres, CUP, Cambridge, p. 401

Hatzes A.P., Vogt S.S., Ramseyer T.F., Misch A., 1996, ApJ 469,808
Jankov S., Foing B.H., 1992, A\&A 256, 533

Joncour I., Bertout C., Menard F., 1994, A\&A 285, L25

King J.R., 1999, PASP 111, 817

Kupka F., Piskunov N.E., Ryabchikova T.A., Stempels H.C., Weiss W.W., 1999, A\&AS 138, 119

Kurucz R.L., 1993, ATLAS-9, CD-ROM \#13

Kürster M., 1993, A\&A 274, 851

Kürster M., Schmitt J.H.M.M., 1990, in Surface Inhomogeneities on Late-Type Stars, Lecture Notes in Physics, Vol. 397, Byrne P.B., D.J. Mullan (eds.). Springer-Verlag, Berlin, p. 69

Lambert D.L., Heath J.E., Lemke M., Drake J., 1996, ApJS 103,183

Piskunov N.E., 1990, in The Sun and Cool Stars: Activity, Magnetism, Dynamos, IAU Colloq. 130, Tuominen I., Moss D., Rüdiger G. (eds.). Springer Verlag, p. 309

Piskunov N.E., Kupka F., Ryabchikova T.A., Weiss W.W., Jeffery C.S., 1995, A\&AS 112, 525

Piskunov N.E., Rice J.B., 1993, PASP 105, 1415

Rice J.B., 1996, in IAU Symp. 176, Stellar Surface Structure, Strassmeier K.G., Linsky J.L. (eds.). Kluwer, Dordrecht, p. 19

Rice J.B., Strassmeier K.G., 1996, A\&A 316, 164 (Paper II)

Rice J.B., Strassmeier K.G., 1998, A\&A 336, 972 (Paper VII)

Rice J.B., Wehlau W.H., Khokhlova V.L., 1989, A\&A 208, 179

Strassmeier K.G., 1996, in IAU Symp. 176, Stellar Surface Structure, Strassmeier K.G., Linsky J.L. (eds.). Kluwer, Dordrecht, p. 289

Strassmeier K.G., 1999, A\&A 347, 225 (Paper XI)

Strassmeier K.G., 2000, in 12th EADN summer school, Selected topics on binary stars, Lecture Notes in Physics. Springer-Verlag (in press)

Strassmeier K.G., Lupinek S., Dempsey R.C., Rice J.B., 1999, A\&A 347, 212 (Paper X)

Strassmeier K.G., Rice J.B., 1998a, A\&A 330, 685 (Paper VI)

Strassmeier K.G., Rice J.B., 1998b, A\&A 339, 497 (Paper IX)

Strassmeier K.G., Rice J.B., 2000, A\&A (in press) (Paper XIV)

Strassmeier K.G., Rice J.B., Wehlau W.H., Vogt S.S., Hatzes A.P., Tuominen I., Piskunov N.E., Hackman T., Poutanen M., 1991, A\&A 247, 130

Strassmeier K.G., Welty A.D., Rice J.B., 1994, A\&A 285, L17

Unruh Y.C., Collier-Cameron A., 1995, MNRAS 273, 116

Unruh Y.C., Collier-Cameron A., 1997, MNRAS 290, L37

Unsöld A., 1955, Physik der Sternatmosphären. Springer Verlag, Berlin, 2nd edition, p. 327

Valenti J.A., Piskunov N.E., 1996, A\&AS 118, 595

Vogt S.S., Penrod G.D., Hatzes A.P., 1987, ApJ 321, 496 